Award Number: W81XWH-09-1-0439

TITLE: Tumorigenic Potential of Transit Amplifying Prostate Cells

PRINCIPAL INVESTIGATOR: Sarki Abba Abdulkadir, M.D., Ph.D.

CONTRACTING ORGANIZATION: Vanderbilt University Nashville, TN 37203-6876

REPORT DATE: June 2012

TYPE OF REPORT: Final

PREPARED FOR: U.S. Army Medical Research and Materiel Command Fort Detrick, Maryland 21702-5012

DISTRIBUTION STATEMENT: Approved for Public Release;

Distribution Unlimited

The views, opinions and/or findings contained in this report are those of the author(s) and should not be construed as an official Department of the Army position, policy or decision unless so designated by other documentation. 


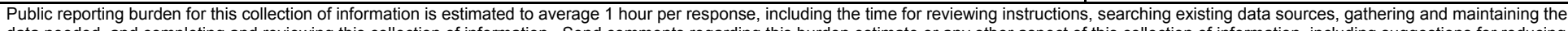

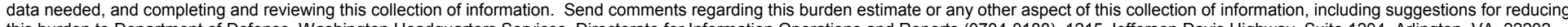

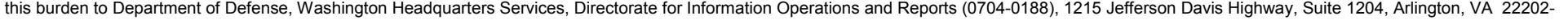

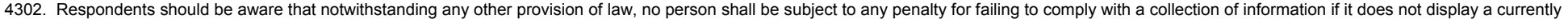
valid OMB control number. PLEASE DO NOT RETURN YOUR FORM TO THE ABOVE ADDRESS.

\begin{tabular}{|l|l|l|l|l}
\hline 1. REPORT DATE & 2. REPORT TYPE & 3. DATES COVERED
\end{tabular}

June 2012 Final

4. TITLE AND SUBTITLE

1 June $2009-31$ May 2012

Tumorigenic Potential of Transit Amplifying Prostate Cells 5a. CONTRACT NUMBER

5b. GRANT NUMBER

W81XWH-09-1-0439

5c. PROGRAM ELEMENT NUMBER

6. AUTHOR(S)

5d. PROJECT NUMBER

Sarki Abba Abdulkadir, M.D., Ph.D.

5e. TASK NUMBER

5f. WORK UNIT NUMBER

E-Mail: sarki.abdulkadir@vanderbilt.edu

7. PERFORMING ORGANIZATION NAME(S) AND ADDRESS(ES)

Vanderbilt University

Nashville, TN 37203-6876

8. PERFORMING ORGANIZATION REPORT NUMBER

9. SPONSORING I MONITORING AGENCY NAME(S) AND ADDRESS(ES)

10. SPONSOR/MONITOR'S ACRONYM(S)

U.S. Army Medical Research and Materiel Command

Fort Detrick, Maryland 21702-5012

11. SPONSOR/MONITOR'S REPORT NUMBER(S)

12. DISTRIBUTION / AVAILABILITY STATEMENT

Approved for Public Release; Distribution Unlimited

\section{SUPPLEMENTARY NOTES}

\section{ABSTRACT}

A better understanding of the identities of the target cells responsible for prostate cancer development and the molecular pathways in these cells are essential for the development of new diagnostic, prognostic and therapeutic approaches. We hypothesized that that loss of the prostate tumor suppressor Nkx3.1 sensitizes prostate cancer epithelial cells to further transformation by arresting cells in a precursor transit amplifying state that is more susceptible to tumorigenesis by oncogenes such as c-MYC. Further, we hypothesized that Nkx3.1 function in prostate cells is regulated by the androgen receptor regulator and pioneer factor FoxA1. Our specific goals were to test the susceptibility of Nkx3.1-null transit amplifying cells to transformation and tumorigenicity in response to MYC and to test the hypothesis that FOXA1 is cofactor involved in the regulation of a subset of NKX3.1 target genes in prostate cells. Using transgenic mice and a prostate regeneration tissue recombination system, we obtained evidence that loss of Nkx3.1 cooperates with MYC expression in promoting prostate tumorigenesis in vivo. We have also obtained evidence that Nkx3.1 preferentially binds near FoxA1 binding sites in prostate cells. Nkx3.1 and FoxA1 form a transcriptional complex involved in the regulation of a cohort of target genes with relevance to prostate tumorigenesis.

15. SUBJECT TERMS

Prostate cancer, stem cell, Nkx3.1 tumor suppressor, MYC oncogene

\begin{tabular}{|c|c|c|c|l|l|}
\hline \multicolumn{2}{|l|}{ 16. SECURITY CLASSIFICATION OF: } & $\begin{array}{l}\text { 17. LIMITATION } \\
\text { OF ABSTRACT }\end{array}$ & $\begin{array}{l}\text { 18. NUMBER } \\
\text { OF PAGES }\end{array}$ & $\begin{array}{l}\text { 19a. NAME OF RESPONSIBLE PERSON } \\
\text { USAMRMC }\end{array}$ \\
\cline { 1 - 2 } $\begin{array}{c}\text { a. REPORT } \\
\text { U }\end{array}$ & $\begin{array}{c}\text { b. ABSTRACT } \\
\text { U }\end{array}$ & $\begin{array}{c}\text { c. THIS PAGE } \\
\text { 19b. TELEPHONE NUMBER (include area } \\
\text { code) }\end{array}$ & UU & 27 \\
\end{tabular}




\section{Table of Contents}

\section{Page}

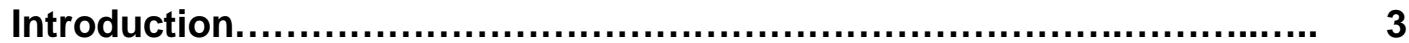

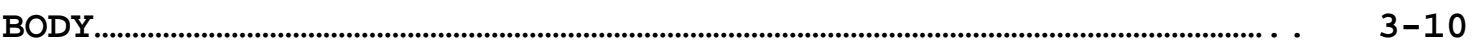

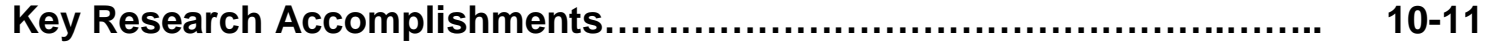

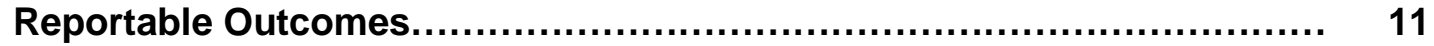

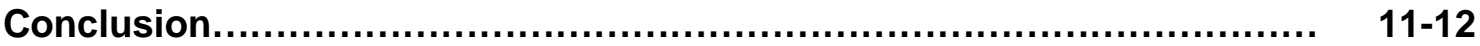

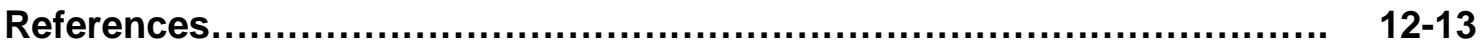

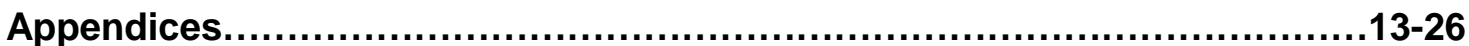




\section{INTRODUCTION}

Detailed knowledge of prostate cancer cells of origin and the molecular pathways important in cellular transformation are essential pre-requisites to the development of novel diagnostic, prognostic and therapeutic approaches $(1,2)$. NKX3.1 is a homeodomain transcription factor whose expression is androgen-dependent and is largely restricted to the luminal epithelial cells of the mature prostate $(3,4)$. NKX3.1 is a tumor suppressor whose expression is lost or attenuated in prostate cancer. Functional studies in transgenic mice suggest that a primary defect in Nkx3.1-null mice is a failure of transit amplifying cells to undergo terminal differentiation and exit the cell cycle in a timely manner $(5,6)$. Loss of Nkx3.1 leads to the dysregulation of many genes (6), but the key targets responsible for. We sought to determine if tumor progression of tumorigenic cells initiated by Nkx3.1 loss requires additional oncogenic events such as Myc overexpression which may promote selfrenewal. Furthermore, the ability of Nkx3.1 to bind and regulate target genes is likely to be modified by additional factors, including so-called pioneer factors that have the ability to bind nucleosomal DNA and destabilize nucleosomes thereby allowing other transcription factors to access their sites (7),(8).

\section{BODY}

Aim 1: To test the susceptibility of Nkx3.1-null transit amplifying cells to transformation and tumorigenicity by MYC in vitro and in vivo using primary and established cell lines. The in vivo studies will use a facile tissue recombination system.

Task 1: Isolate prostate epithelial cells from Nkx3.1+/+ and Nkx3.1-/- mice, infect with MYCexpressing or GFP control lentivirus. Generate and analyze numbers and sizes of prostaspheres formed by growth in matrigel. Serially passage prostaspheres by trypsin digestion; assess rate of formation of secondary and tertiary spheres in matrigel as an indication of self-renewal potential.

Task 2: Molecular characterization of prostaspheres using RT-PCR and immunohistochemistry for markers of differentiation. In vitro assays of transformation on lentivirus-infected mouse primary prostate epithelial cells: focus formation and soft agar assays.

Task 3: In vivo tissue recombination experiments. Primary mouse prostate epithelial cells from Nkx3.1+/+ and Nkx3.1-/- mice isolated and infected with MYC and GFP-control lentiviruses. Cells are recombined with fetal rat urogenital mesenchyme and implanted in severe combined immunodeficient mice. 
Task 1-3 Results: We established prostaspheres in matrigel from primary prostate cells isolated from wild type and Nkx3.1 knockout mice. The sphere cells express the prostate epithelial marker keratin 8, K8. Quantitation of primary sphere number and size did not show a significant difference between wild type and

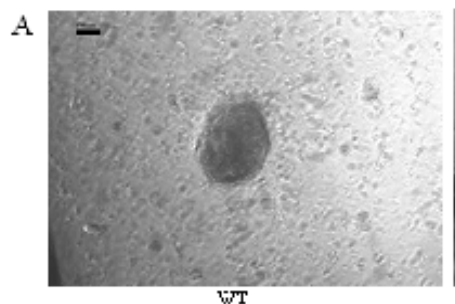

$\mathbf{B}$

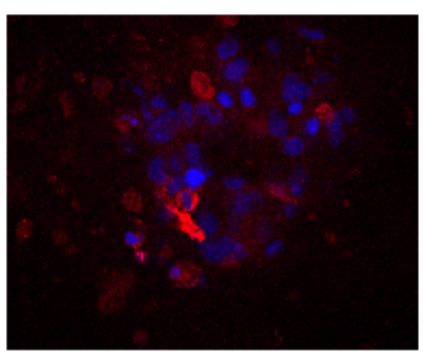

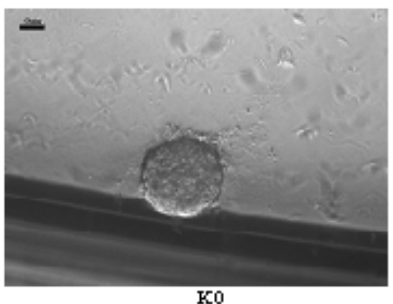

Fig. 1: Prostaspheres from Nkx3.1 wild type (WT) and knockout mice (KO). A) Primary prostate epithelial cells from $8 w k$ old mice of the indicated genotypes were grown in matrigel and images captured after 10 days. B) Fluorescence confocal microscopy shows that these spheres express luminal epithelial marker K8. (Nucleus -blue, K8 - red) knockout (Fig 1). Secondary spheres generated by serial passaging show a trend for reduced number of spheres from the Nkx3.1 knockout mice. However, gene expression analysis reveals that spheres lack expression of $\mathrm{Nkx3.1}$. We isolated adult prostate epithelial cells from adult Nkx3.1+/+ and Nkx3.1-/mice and infected them with lentivirus co-expressing MYC and YFP (yellow fluorescent protein) or YFP alone. The prostate epithelial cells were then recombined with fetal rat urogenital mesenchyme (UGM) in collagen and implanted under the kidney capsules of immunodeficient mice to regenerate prostates). Six weeks later, the grafts were harvested and characterized by histology. Nkx3.1+/+;YFP grafts formed normal looking prostate glands, while Nkx3.1-/;YFP grafts showed prostatic epithelial hyperplasia consistent with the phenotype of Nkx3.1-/- mice. Nkx3.1+/+;MYC grafts on the other hand consisted of glands containing high-grade PIN (HGPIN) lesions while
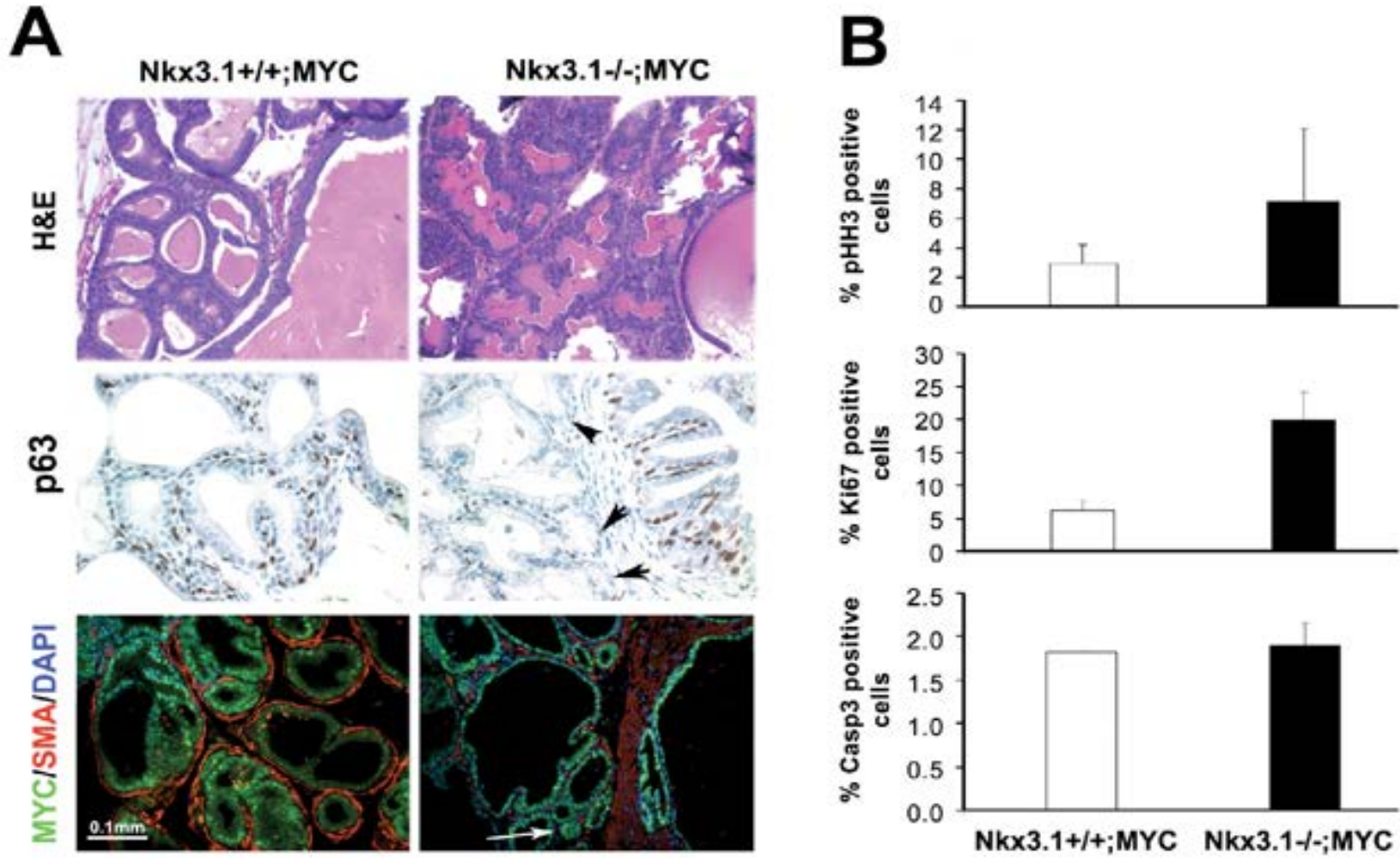

Fig. 2: Cooperativity between MYC and Nkx3.1-loss in vivo by prostate regeneration. A, Histology, focal loss of basal cells (p63) and focal loss of smooth muscle actin, SMA indicate progression in Nkx3.1-/-;MYC prostate grafts compared to Nkx3.1+/+;MYC grafts. B, Increased proliferation (phospho-histone H3, pHH3 and Ki67 staining) in Nkx3.1-/-; MYC grafts compared to Nkx3.1+/+;MYC grafts. Double staining with MYC was used to enable quantitation of proliferation in MYCexpressing glands. Apoptosis (activated- caspase 3 staining) was unchanged. 
Nkx3.1-/-;MYC grafts showed HGPIN with microinvasion as confirmed by focal loss of smooth muscle actin as well as p63 basal cell staining (Fig. 2). The Nkx3.1-/-;MYC grafts also showed higher proliferation without a concomitant increase in apoptosis. We obtained similar results by tissue recombination of transgenic MYC+;Nkx3.1-deficient prostates (9). MYC+;Nkx3.1 prostates contained more foci of PIN and microinvasive cancer with higher proliferation. (All differences significant with $\mathrm{P}<0.05$ ).

Difficulties encountered and response: Our gene expression analyses showed that when prostate cells are grown as spheres, they lose expression of Nkx3.1. This precluded functional analysis comparing Nk3.1+/+ and Nkx3.1-/- mouse-derived spheres and further emphasized the need for in vivo analysis, which we completed.

Aim 2: To test the hypothesis that FOXA1 is cofactor involved in the regulation of a subset of NKX3.1 target genes in prostate cells. We will determine whether FOXA1 is required as a "pioneer factor" to allow NKX3.1 to bind to chromatin and regulate these target genes. The role of histone modifications, specifically histone H3 methylation on FOXA1 and NKX3.1 binding to target genes will be assessed. Finally, we will examine the functional significance of selected NKX3.1/FOXA1-regulated genes in prostate tumorigenesis.

Task 4: Use siRNA to knockdown FOXA1, NKX3.1 or both in LNCaP cells, then examine target gene expression for 20 selected NKX3.1/FOXA1 targets by qRT-PCR. FOXA1. Assess if FOXA1 knockdown globally affects binding of NKX3.1 to chromatin, and vice versa.

Task 5: Examine if NKX3.1 interacts with FOXA1 using co-immunoprecipitation. Map interaction domains. Examine interaction on chromatin by ChIP-re-ChIP analysis.

Task 6: Examine changes in H3K4me1/2 following FOXA1 or NKX3.1 knockdown. Overexpress the lysine demethylase KDM1, and then assess H3K4me1/2 status and FOXA1 and NKX3.1 binding by ChIP.

Task 7: Determine the correlation between the occurrence of NKX3.1/FOXA1 binding sites within $20 \mathrm{~Kb}$ of the TSS of genes and the co-expression of the genes with FOXA1 and NKX3.1, using published databases of prostate cancer microarray studies.

Results: Tasks 4-7: Analysis of genomic NKX3.1 binding sites obtained by ChIP-Seq showed that in both the human prostate cell line LNCaP and in mouse prostate, NKX3.1 bound DNA fragments are significantly enriched in FOXA consensus sequences (Fig 3). Other enriched motifs apart from the NKX3.1 consensus itself include AR/GR and GATA motifs. Notably, the NKX3.1, FOXA and AR/GR motifs are all enriched near the peak summits as shown by the violin plots in Fig 3. By contrast, a negative control motif for RUNX2 was depleted near the peak summits.

As FOXA1 is known to promote prostate tumor progression and to represent a novel hallmark of castration-resistant prostate cancer (10), we examined potential interaction between FOXA1 and NKX3.1. We found evidence of physical interaction between NKX3.1 and FOXA1 by co-immunoprecipitation (Fig 4). Furthermore, interaction occurs even in the presence of ethidium bromide, indicating that binding is 
independent of DNA. We also confirmed the reported interaction between AR and NKX3.1 (Fig 4). We mapped interaction of NKX3.1 to the forkhead domain of FOXA1 using a series of deletion mutants (Fig 4). The forkhead domain of FOXA1 is essential for interaction with NKX3.1.

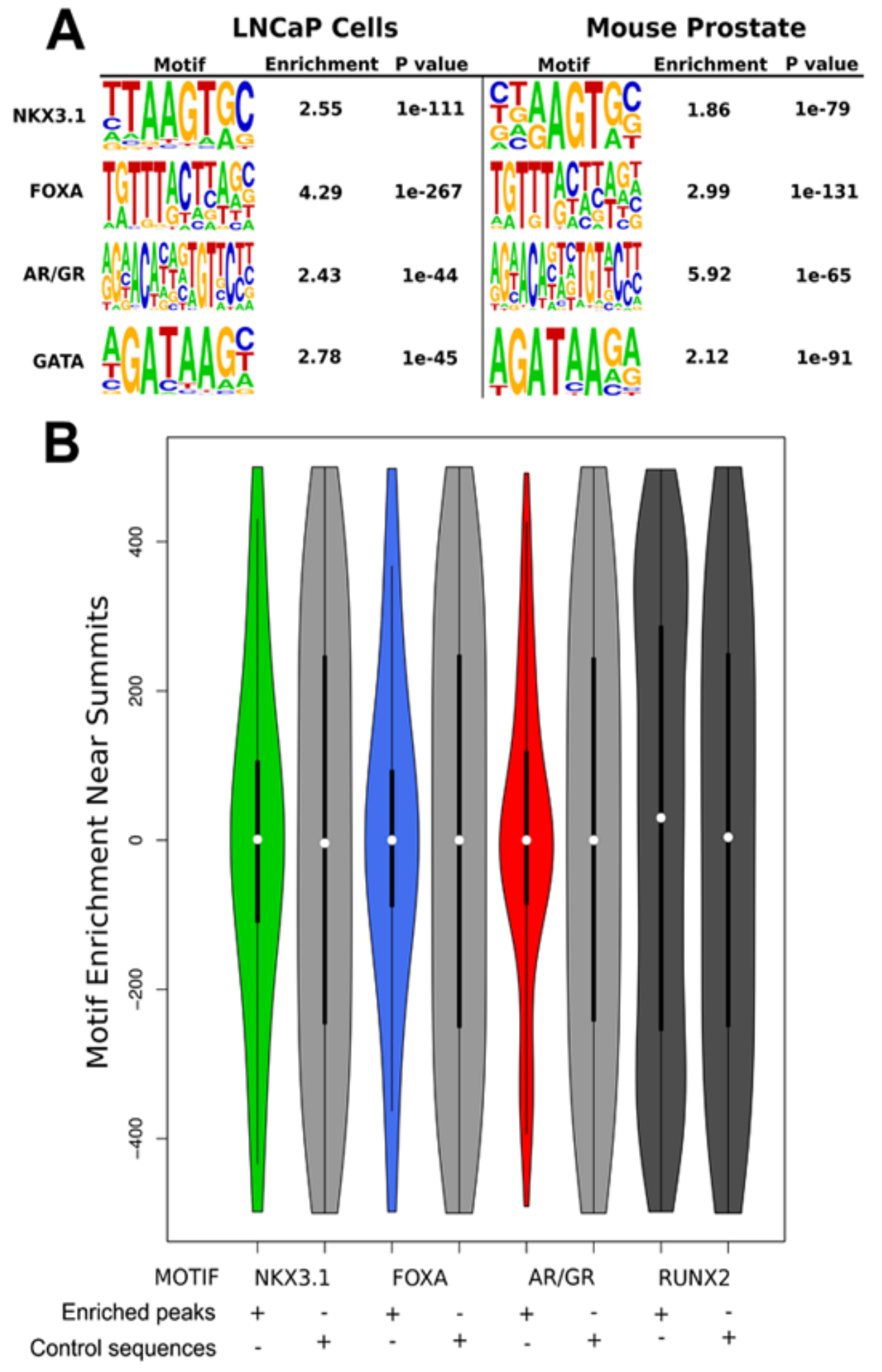

Analsyis of ChIP-Seq data in LNCaP cells for AR, FOXA1 and NKX3.1 allowed us to determine sites and genes bound by all combinations of the 3 transcription factors (Fig. 5). Notably, 1800 genes were bound by all 3 factors, while 2664 genes were uniquely bound by NKX3.1. These results suggest that while NKX3.1 may be involved in coregulating genes in concert with AR/FOXA1, it may also have a unique transcriptional agenda in prostate cells. Indeed gene ontology analysis (Fig 6) shows that genes bound by NKX3.1 and AR/FOXA1 regulate similar pathways e.g. glypican synthesis, TGF beta, TNF and TRAIL signaling. However genes uniquely bound by NKX3.1 are involved in gene transcription and splicing.

Fig 3: A and B: Motif analysis of NKX3.1-bound sequences from ChIP-Seq shows enrichment for FOXA, AR/GR and GATA motifs. 
A
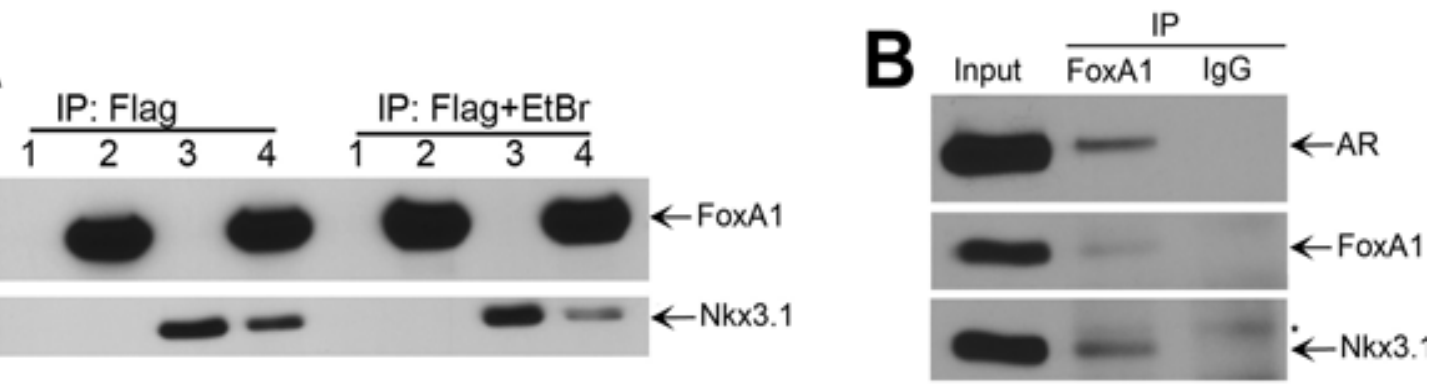

C

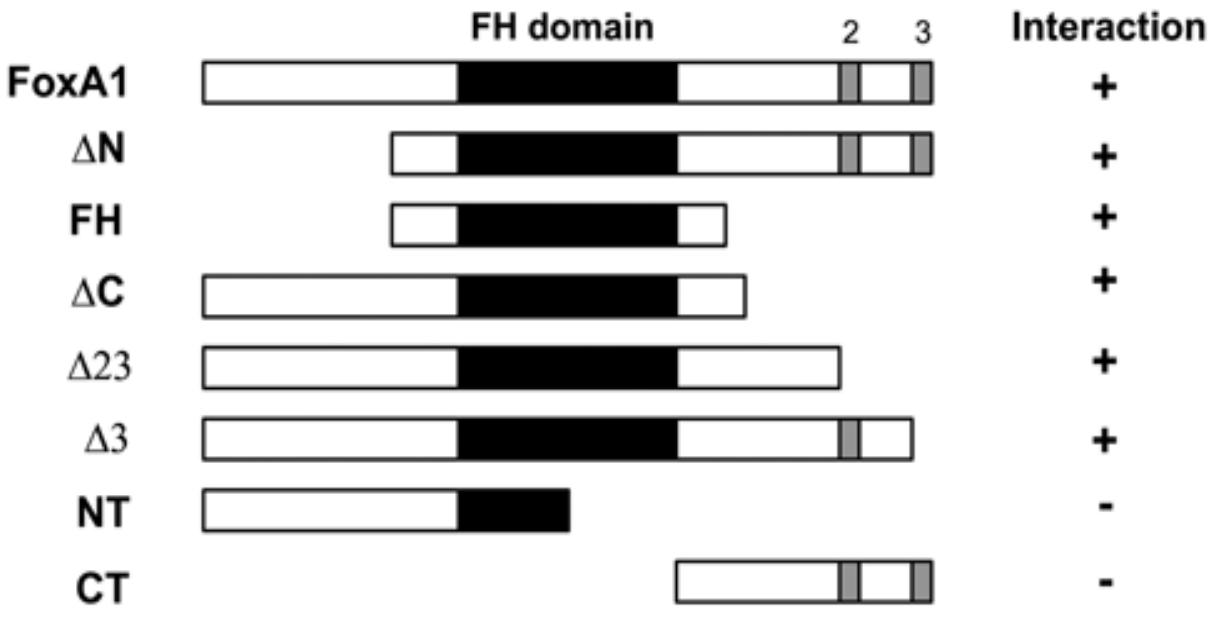

D

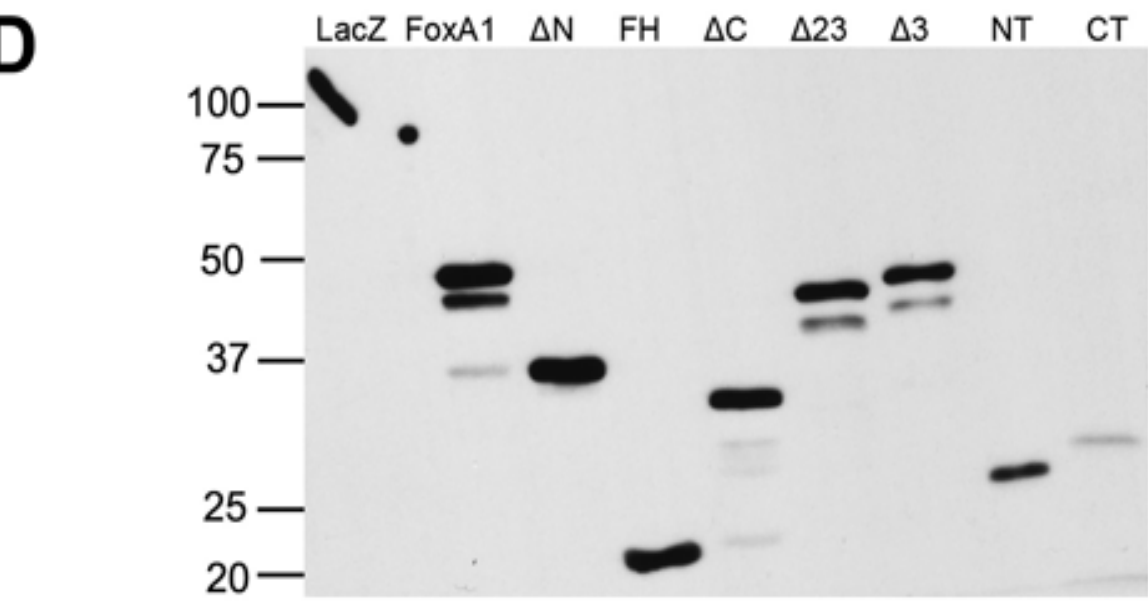

IB: V5

$\mathbf{E}$

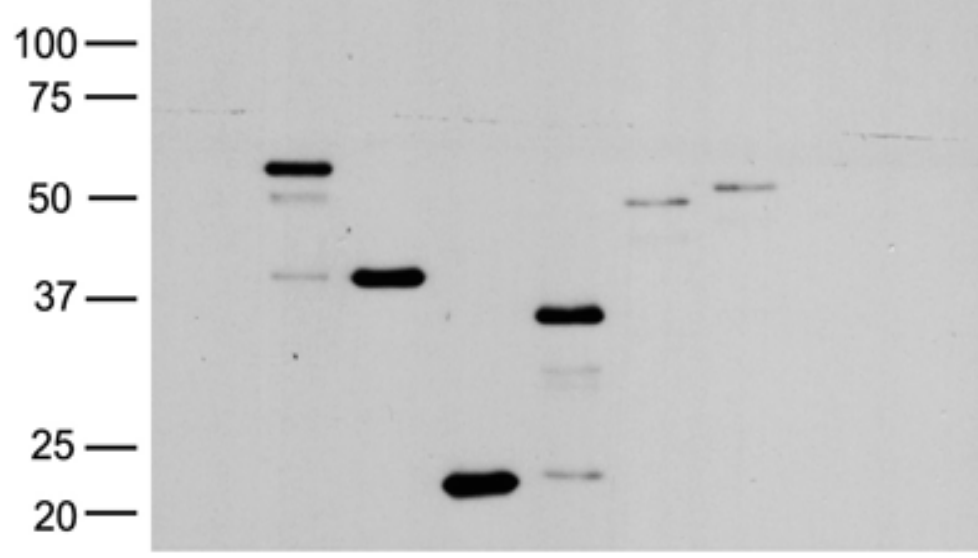

GST-Nkx3.1

IB: V5

Fig 4: A, Co-immunoprecipitation shows binidng of NKX3.1 to FOXA1 even in the presence of EtBr in transfecetd 293 cells. B, NKX3.1 interacts with FOXA1 and AR in LNCaP cells. C-E, FOXA1 deeltion mutants used to map NKX3.1 interaction site by GST pulldown assay. 
Binding sites

AR NKX3-1

3,009

41,256

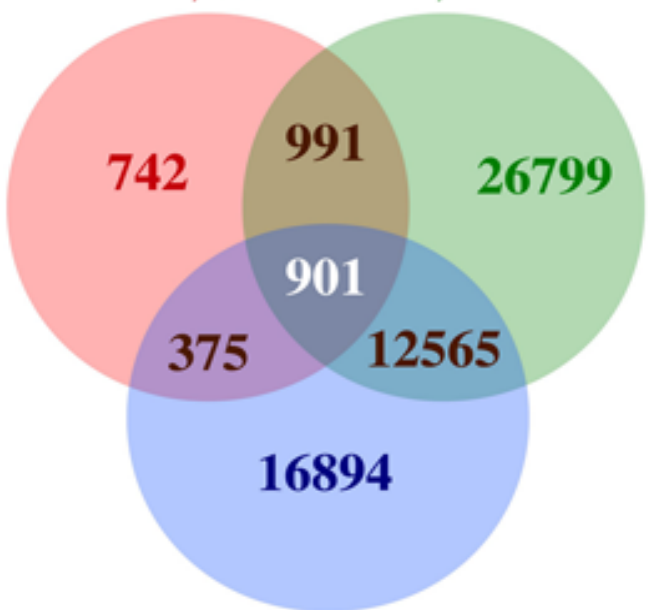

FOXA1

30,735
$B$ Genes bound

AR NKX3-1

$2,107 \quad 10,506$

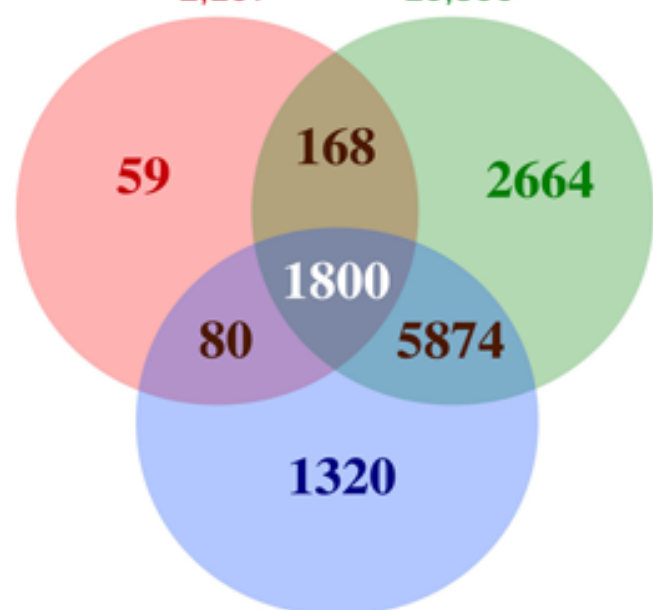

FOXA1

9,074

Fig 5: Venn diagran showing overlap of binding sites (A) and genes (B) bound by NKX3.1, FOXA1 and AR.

Pathway
Tene Expression

Fig 6: Gene Ontology analysis for genes bound NKX3.1 alone, NKX3.1 and FOXA1, or NKX3.1, FOXA1 and AR 
To examine the functional consequences of depleting FOXA1 on NKX3.1 chromatin binding and vice versa, we established LNCaP cells with knockdown of these factors (Fig 7). These cells have been processed by ChIP and the samples submitted for deep sequencing. We are awaiting the data to complete analysis.

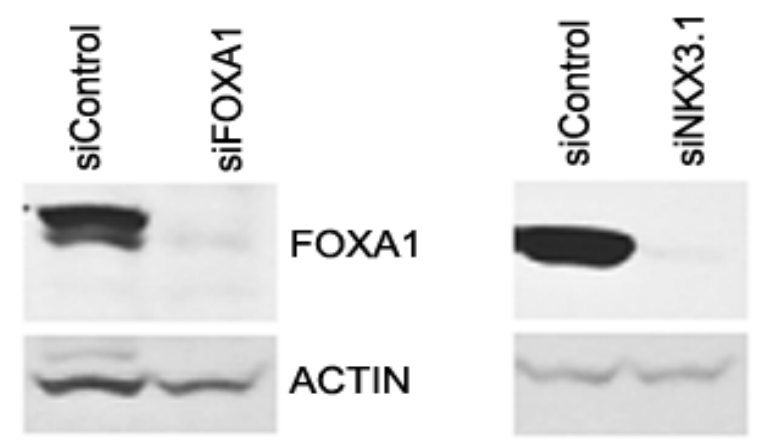

NKX3.1

We examined the relationship between H3 methylation, NKX3.1, FOXA1 and AR binding by integrating NKX3.1, FOXA1, AR, H3K4me2 and H3K4me3 ChIP-Seq data in LNCaP cells. We identified ACTIN binding of NKX3.1 enhancers identified by H3K4me2/3, in many cases also Fig 7: LNCaP cells with knockdown of FOXA1 and NKX3.1. showing co-occupation by AR and FOXA1 while other NKX3.1 sites lack methylation marks, AR and FOXA1 (Fig 8). The latter may represent non-functional sites. After FOXA1 knockdown by siRNA, some AR sites are retained or lost; however some new sites appear (Fig 9), consistent with recent findings (11, 12). These findings indicate that FOXA1 modulates AR binding to chromatin. We are currently completing the analysis of NKX3.1 binding after FOXA1 knockdown to determine whether FOXA1 modulates NKX3.1 binding in a similar manner.

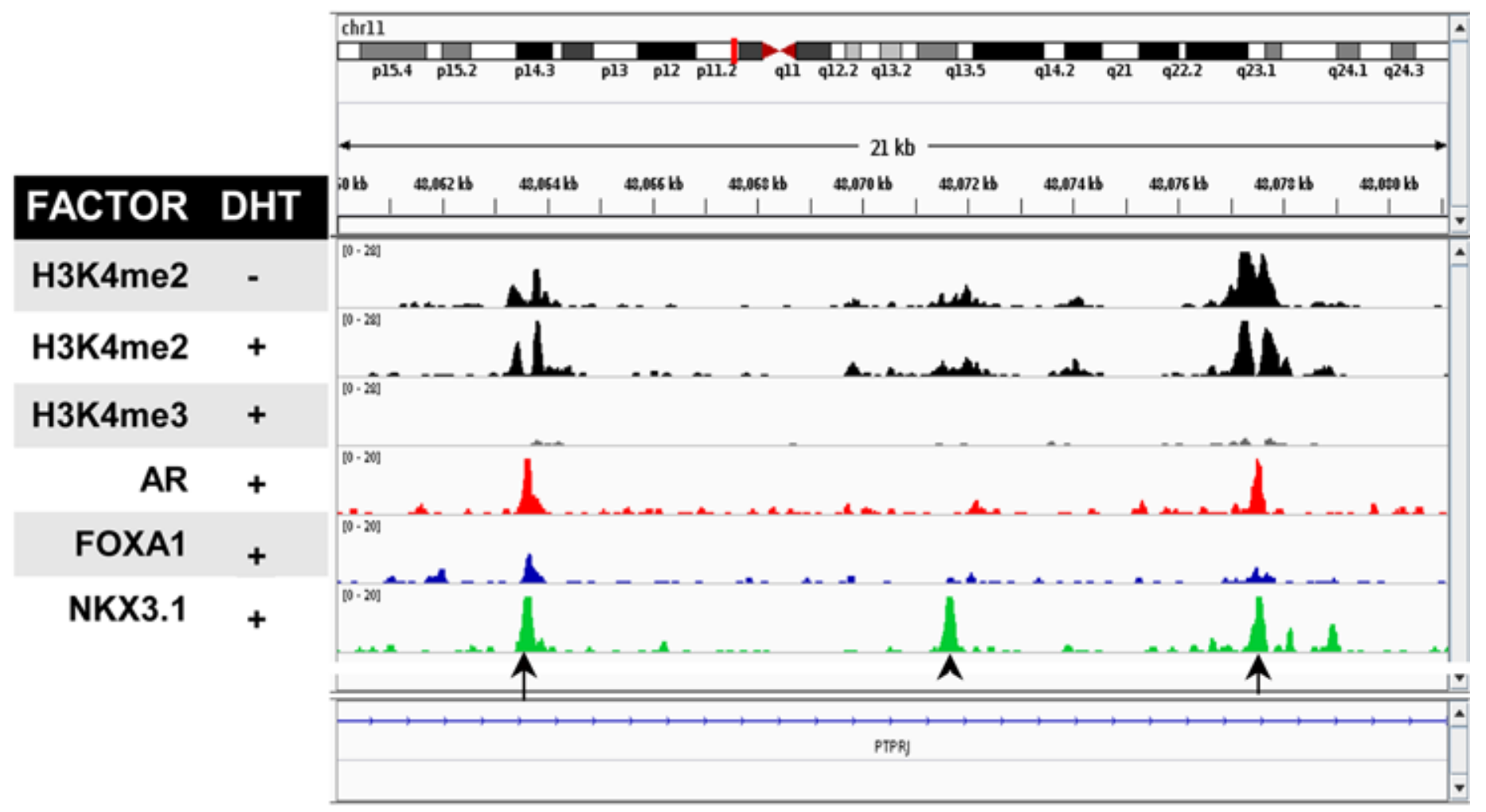

Fig 8: Examples of NKX3.1 sites that overlap with H3K4me2/3, AR and FOXA1 (arrows) and one that does not (arrwohead). 


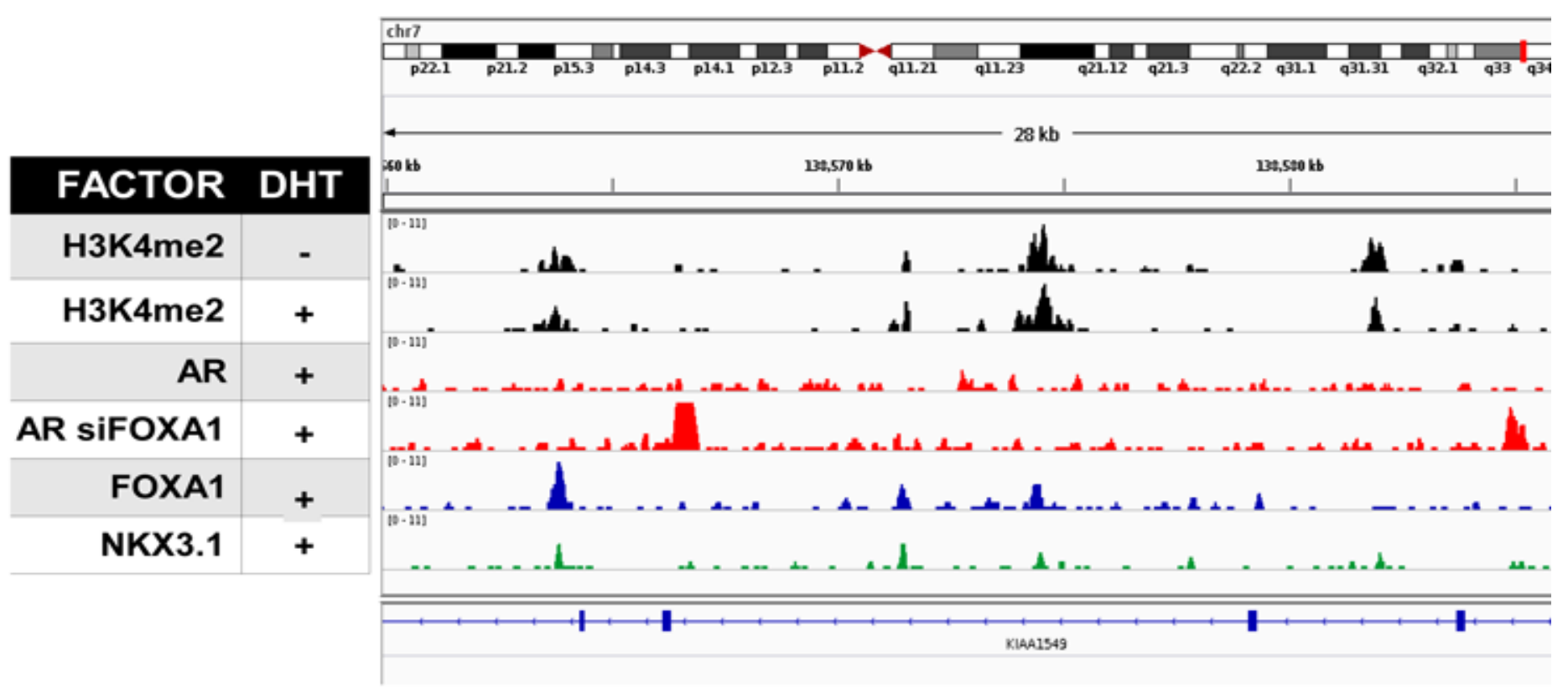

Fig 9: Appearance of new AR sites after knockdown fo FOXA1 by siRNA. Part of Figures 8 and 9 generated by re-analysis of datasets from (12).

Difficulties encountered and response: These mainly relate to the longer time it has taken for our ChIP samples to be sequenced by the core facility. We still have FOXA1 and AR ChIP samples in NKX3.1-depeleted cells that are being sequenced. The analysis of the sequence data will be completed shortly. We anticipate publishing two manuscripts reporting the studies in Aim 2.

\section{KEY RESEARCH ACCOMPLISHMENTS}

- Establishment of prostate sphere assays using matrigel, using wild type and Nkx3.1 knockout primary prostate cells

- Analysis of gene expression in prostate sphere cells, demonstrating that NKX3.1 is lost in cells grown as spheres

- Demonstration that loss of Nkx3.1 cooperates with MYC expression in prostate tumorigenesis in vivo using prostate regeneration by tissue recombination

- Identification of enrichment of FOXA consensus motif in NKX3.1-bound ChIP-Seq DNA fragments. Subsequent demonstration that FOXA1 frequently binds close to NKX3.1 at many sites in prostate cells in vivo

- Demonstration that NKX3.1 and FOXA1 interact and mapping the NKX3.1 interacting site in FOXA1 to the forkhead domain

- Mapping of NKX3.1, FOXA1 and AR binding sites in prostate cells and relation to histone methylation marks for enhancers 
- Identification of a set of genes uniquely regulated by NKX3.1 in prostate cells and the finding that these genes are involved in the regulation gene expression. This suggests a unique role for NKX3.1 in prostate epithelial cells.

\section{REPORTABLE OUTCOMES:}

The following publications and abstract have been supported in whole or in part by the grant:

Anderson PD*, McKissic SA*, Logan M, Roh M, Franco OE, Wang J, Doubinskaia I, van der Meer R, Hayward SW, Eischen CM, Eltoum I, Abdulkadir SA. Nkx3.1 and c-Myc cross-regulate shared target genes in mouse and human prostate tumorigenesis. J Clin Invest 2012 May 1;122(5):1907-19. doi: 10.1172/JCI58540. Epub 2012 Apr 9. [*co-first authors]

Philip D. Anderson, Sydika A. McKissic, Monica Logan, Meejeon Roh, Omar Franco, Jie Wang, Irina Doubinskaia, Riet van der Meer, Simon Hayward, Christine Eischen, Isam-Eldin A. Eltoum, and Sarki A. Abdulkadir. Abstract 2983: Nkx3.1 and c-Myc co-regulate shared target genes involved in prostate cancer AACR 2012 Annual Meeting. Poster Presentations - Bioinformatics Applications and Gene Expression Analysis

Sydika A. McKissic and Sarki A. Abdulkadir. Abstract 2401 Loss of Nkx3.1 cooperates with Myc overexpression to promote prostate tumorigenesis. AACR 2011 Annual Meeting

Data related to this project were reported at the 2011 IMPACT Meeting in Florida.

Individuals funded by the award mechanism:

Philip Anderson, PhD, postdoctoral fellow

Sydika McKissic, graduate student

Jie Wang, PhD, postdoctoral fellow

\section{CONCLUSION:}

This funding period has been quite productive. The project has been instrumental in enabling us to establish prostate sphere assays for primary mouse prostate epithelial cells and to develop a tissue recombination prostate regeneration model showing that loss of Nkx3.1 cooperates with Myc overexpression to promote prostate tumorigenesis. Interestingly, parallel studies in our lab on Nkx3.1 target genes converged nicely with the tumorigenesis studies because we found that Nkx3.1 shares many targets with Myc. This was unexpected but 
allowed us to integrate the Nkx3.1/Myc tumorigenesis studies with the Nkx3.1/Myc target genes studies in the JCI paper listed above.

In the second part of the studies, we focused on examining the possible role of FOXA1 as a cofactor in the regulation of NKX3.1 target genes. As a pioneer factor able to remodel nucleosomes and "open” chromatin, FOXA1 may be required to allow NKX3.1 to access its DNA binding sites in chromatin. Consistent with this hypothesis, we have so far shown that NKX3.1 and FOXA1 bind to DNA sites that are very close to each other in the genome. Furthermore, FOXA1 can physically interact with NKX3.1. However, the relationship between FOXA1 and NKX3.1 may well be more complicated, similar to the relationship between FOXA1 and AR. While depletion of FOXA1 in prostate cells abrogates AR binding to some of its sites, a large number of apparently functional AR sites are exposed in the absence of FOXA1. We are completing analysis of studies examining this issue with relation to NKX3.1.

The recent explosion of genome-wide binding data in LNCaP cells for several transcription factors and histone modifications allowed us to integrate our NKX3.1 binding data with binding data for FOXA1, AR and for histone H3 methylation patterns in the presence and absence of androgen and before and after FOXA1 depletion. NKX3.1 binding sites demonstrate distinct patterns, in which some sites co-localize with AR, FOXA1 and enhancer histone methylation marks while other NKX3.1 binding sites occur in the absence of these signals.

Overall, these findings have provided us key insights into prostate tumor initiation by loss NKX3.1 and cooperation with c-MYC. Loss of NKX3.1 sensitizes cells to transformation by MYC, in part by the two factors cross-regulating the same target genes. They have also raised new questions about how NKX3.1 interactions with AR and FOXA1 are integrated with histone H3 methylation to modulate target gene expression that should spur further studies.

\section{REFERENCES}

1. Lawson, D.A., and Witte, O.N. 2007. Stem cells in prostate cancer initiation and progression. J Clin Invest 117:2044-2050.

2. $\quad$ Dirks, P.B. 2008. Cancer's source in the peripheral nervous system. Nat Med 14:373-375.

3. Abdulkadir, S.A. 2005. Mechanisms of prostate tumorigenesis: roles for transcription factors Nkx3.1 and Egr1. Ann N Y Acad Sci 1059:33-40.

4. Abate-Shen, C., and Shen, M.M. 2000. Molecular genetics of prostate cancer. Genes Dev 14:2410-2434.

5. $\quad$ Abdulkadir, S.A., Magee, J.A., Peters, T.J., Kaleem, Z., Naughton, C.K., Humphrey, P.A., and Milbrandt, J. 2002. Conditional loss of nkx3.1 in adult mice induces prostatic intraepithelial neoplasia. Mol Cell Biol 22:1495-1503.

6. Magee, J.A., Abdulkadir, S.A., and Milbrandt, J. 2003. Haploinsufficiency at the Nkx3.1 locus. A paradigm for stochastic, dosage-sensitive gene regulation during tumor initiation. Cancer Cell 3:273283.

7. Zaret, K. 1999. Developmental competence of the gut endoderm: genetic potentiation by GATA and HNF3/fork head proteins. Dev Biol 209:1-10. 
8. Zaret, K.S., Watts, J., Xu, J., Wandzioch, E., Smale, S.T., and Sekiya, T. 2008. Pioneer factors, genetic competence, and inductive signaling: programming liver and pancreas progenitors from the endoderm. Cold Spring Harb Symp Quant Biol 73:119-126.

9. Anderson, P.D., McKissic, S.A., Logan, M., Roh, M., Franco, O.E., Wang, J., Doubinskaia, I., van der Meer, R., Hayward, S.W., Eischen, C.M., et al. 2012. Nkx3.1 and Myc crossregulate shared target genes in mouse and human prostate tumorigenesis. J Clin Invest 122:1907-1919.

10. Gerhardt, J., Montani, M., Wild, P., Beer, M., Huber, F., Hermanns, T., Muntener, M., and Kristiansen, G. 2012. FOXA1 promotes tumor progression in prostate cancer and represents a novel hallmark of castration-resistant prostate cancer. Am J Pathol 180:848-861.

11. Sahu, B., Laakso, M., Ovaska, K., Mirtti, T., Lundin, J., Rannikko, A., Sankila, A., Turunen, J.P., Lundin, M., Konsti, J., et al. 2011. Dual role of FoxA1 in androgen receptor binding to chromatin, androgen signalling and prostate cancer. Embo J 30:3962-3976.

12. Wang, D., Garcia-Bassets, I., Benner, C., Li, W., Su, X., Zhou, Y., Qiu, J., Liu, W., Kaikkonen, M.U., Ohgi, K.A., et al. 2011. Reprogramming transcription by distinct classes of enhancers functionally defined by eRNA. Nature 474:390-394.

\section{APPENDICES}




\title{
Nkx3.1 and Myc crossregulate shared target genes in mouse and human prostate tumorigenesis
}

\author{
Philip D. Anderson,, ${ }^{1}$ Sydika A. McKissic, ${ }^{1}$ Monica Logan, ${ }^{2}$ Meejeon Roh,, ${ }^{1}$ Omar E. Franco, ${ }^{3}$ \\ Jie Wang, ${ }^{1}$ Irina Doubinskaia, ${ }^{1}$ Riet van der Meer, ${ }^{1}$ Simon W. Hayward,3,4 \\ Christine M. Eischen, ${ }^{1,4}$ Isam-Eldin Eltoum, ${ }^{5}$ and Sarki A. Abdulkadir ${ }^{1,4}$

\begin{abstract}
${ }^{1}$ Department of Pathology, Microbiology and Immunology, Vanderbilt University Medical Center, Nashville, Tennessee, USA. 2Department of Biochemistry and Cancer Biology, Meharry Medical College, Nashville, Tennessee, USA. ${ }^{3}$ Department of Urology and ${ }^{4}$ Department of Cancer Biology, Vanderbilt University Medical Center, Nashville, Tennessee, USA. ${ }^{5}$ Department of Pathology, University of Alabama at Birmingham, Birmingham, Alabama, USA.
\end{abstract}

\begin{abstract}
Cooperativity between oncogenic mutations is recognized as a fundamental feature of malignant transformation, and it may be mediated by synergistic regulation of the expression of pro- and antitumorigenic target genes. However, the mechanisms by which oncogenes and tumor suppressors coregulate downstream targets and pathways remain largely unknown. Here, we used ChIP coupled to massively parallel sequencing (ChIP-seq) and gene expression profiling in mouse prostates to identify direct targets of the tumor suppressor $\mathrm{Nkx3.1}$. Further analysis indicated that a substantial fraction of $\mathrm{Nkx} 3.1$ target genes are also direct targets of the oncoprotein Myc. We also showed that $\mathrm{Nkx3.1}$ and Myc bound to and crossregulated shared target genes in mouse and human prostate epithelial cells and that $\mathrm{Nkx3.1}$ could oppose the transcriptional activity of Myc. Furthermore, loss of $\mathrm{Nkx3.1}$ cooperated with concurrent overexpression of $\mathrm{Myc}$ to promote prostate cancer in transgenic mice. In human prostate cancer patients, dysregulation of shared NKX3.1/MYC target genes was associated with disease relapse. Our results indicate that NKX3.1 and MYC coregulate prostate tumorigenesis by converging on, and crossregulating, a common set of target genes. We propose that coregulation of target gene expression by oncogenic/tumor suppressor transcription factors may represent a general mechanism underlying the cooperativity of oncogenic mutations during tumorigenesis.
\end{abstract}

\section{Introduction}

Classic studies showed that malignant transformation is crucially dependent on cooperative interactions between distinct oncogenic mutations $(1,2)$. In a model of Ras/p53-mediated transformation, cooperativity between oncogenic mutations was shown to be mediated by the synergistic coregulation of a class of targets called cooperation response genes, which are important for tumorigenicity (3). Presently, it is unknown whether coregulation of multiple cancer-related target genes by cooperating oncogenic mutations is a generalized process in carcinogenesis (4). Furthermore, the potential mechanism or mechanisms by which alterations in specific oncogenes/tumor suppressor genes (TSGs) may lead to a cooperative response in target gene expression are not clear. In principle, for oncogenes/TSGs that encode transcription factors, direct coregulation of target gene expression might provide a mechanistic explanation for cooperativity. Here, we present our analysis of genome-wide binding sites for the prostate tumor suppressor and transcriptional regulator Nkx3.1, which led us to discover an interaction between Nkx3.1 and the oncoprotein Myc. We show that Nkx3.1 and Myc directly regulate a set of shared target genes and cooperate in prostate tumorigenesis.

$\mathrm{Nkx} 3.1$ is a homeodomain-containing transcription factor with roles in prostate development and cancer (5). Recent studies indicate that Nkx3.1 marks a population of castration-resistant luminal

Authorship note: Philip D. Anderson and Sydika A. McKissic contributed equally to this work.

Conflict of interest: The authors have declared that no conflict of interest exists. Citation for this article: J Clin Invest. 2012;122(5):1907-1919. doi:10.1172/JCI58540. stem cells and is required for stem cell self renewal (6). Nkx3.1 protein is commonly attenuated in mouse and human prostate tumors $(7,8)$, and a germ line sequence variant that impairs NKX3.1 DNA binding has been associated with hereditary prostate cancer (9). More recently, Genome-Wide Association Studies (GWAS) identified a functional variant in NKX3.1 associated with reduced gene expression as a risk factor for sporadic prostate cancer (10-12). Deletion of one or both alleles of $N k \times 3.1$ in mice leads to epithelial hyperplasia, dysplasia, and low-grade prostatic intraepithelial neoplasia (LGPIN) $(13,14)$. Introduction of additional mutations, such as deletion of a single allele of the Pten TSG, promotes the progression of the premalignant lesions in $N k \times 3.1^{-/-}$and $N k \times 3.1^{+/-}$ mice to invasive carcinoma with metastasis $(15,16)$. Conversely, in a conditional Pten-deletion model of prostate cancer, Nkx3.1 expression was uniformly lost, and reconstituting Nkx3.1 expression by lentiviral-mediated gene transfer into Pten-deficient cells potently suppressed tumorigenicity $(17,18)$. Thus, a wealth of evidence suggests that reductions in the amount of Nkx3.1 protein may be permissive for prostate cell transformation.

Despite the critical role played by Nkx3.1 in prostate development and cancer, we know very little about the sites bound by this transcription factor in vivo or the genes and pathways it regulates. To obtain a comprehensive view of the genes and pathways regulated by Nkx3.1 in the prostate, we employed ChIP coupled with massively parallel sequencing (ChIP-seq) to identify Nkx3.1-binding sites across the genome (the Nkx3.1 "cistrome"). We integrated the ChIP-seq data with gene expression profiling to identify "direct" Nkx3.1 target genes that are both bound and regulated 
A

Nkx3.1 ChIP-seq
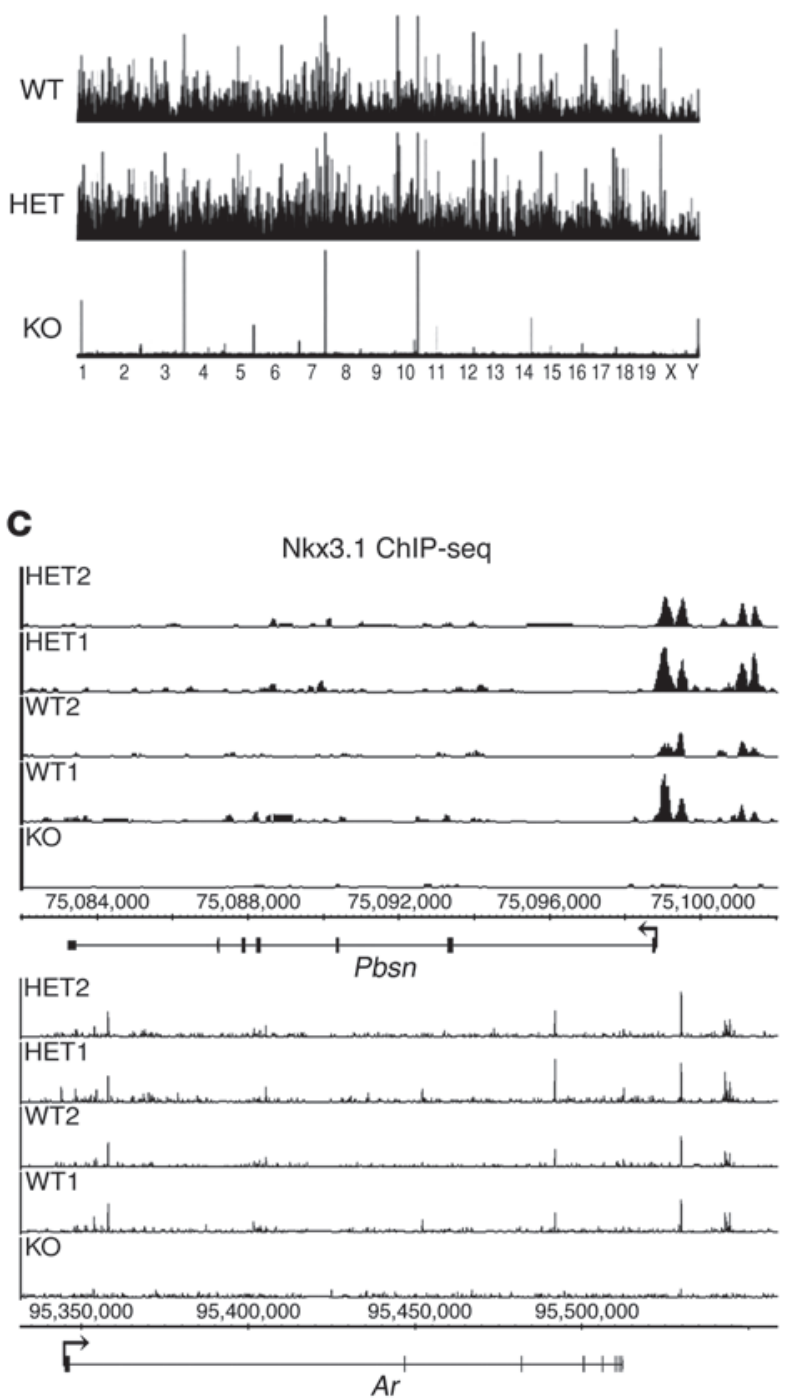

B
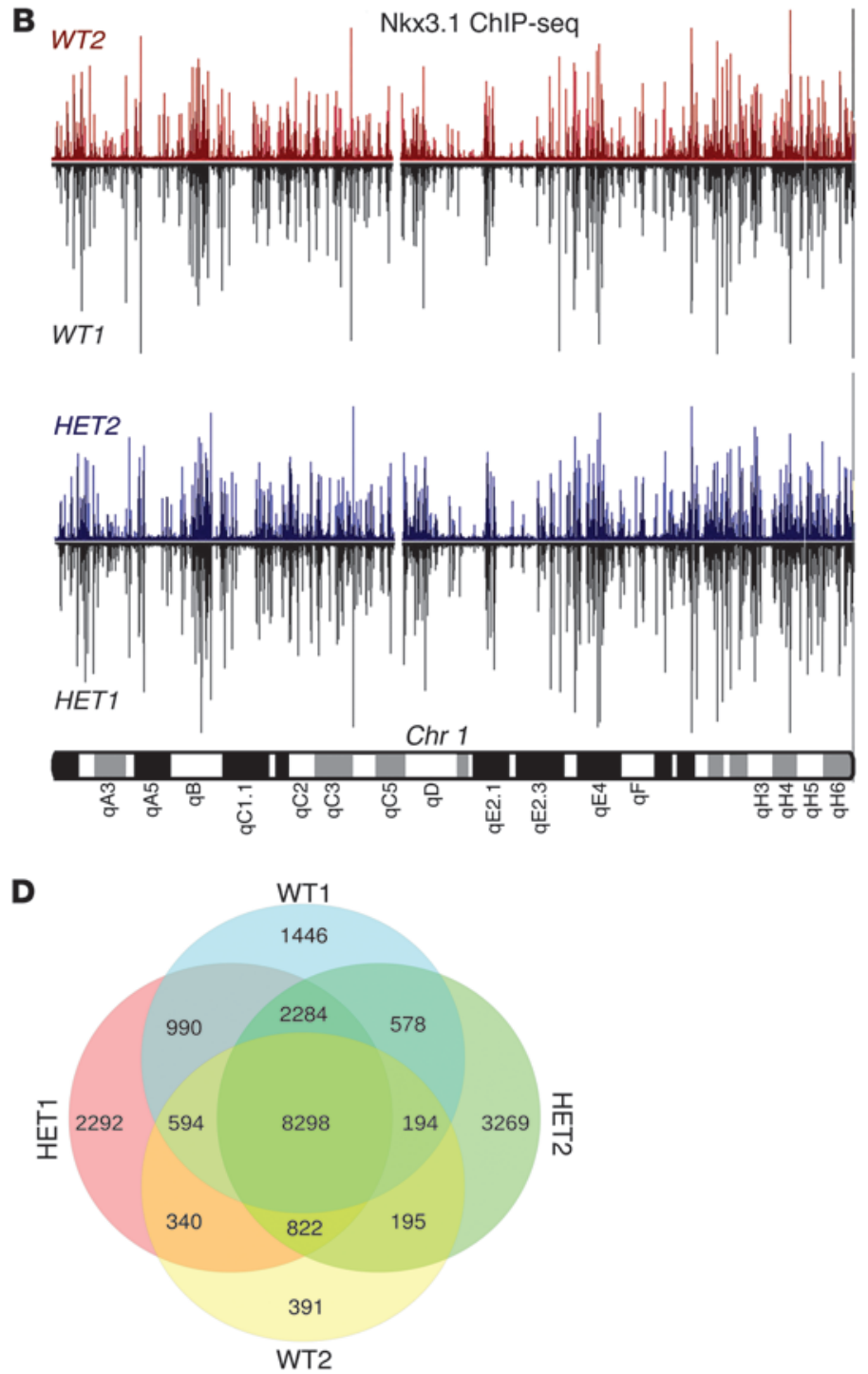

Figure 1

Analysis of genome-wide Nkx3.1-binding sites in the mouse prostate. (A) Visualization of Nkx3.1-binding sites across all mouse chromosomes in $N k \times 3.1^{+/+}(\mathrm{WT}), N k \times 3.1^{+/-}(\mathrm{HET})$, and $N k \times 3.1^{-/-}(\mathrm{KO})$ mouse prostates. Not to scale. (B) High reproducibility in Nkx3.1-binding sites among biological replicates and genotypes. Chromosome 1 is shown as an example. (C) Integrated Genome Browser (IGB) shots showing Nkx3.1binding sites near the Pbsn and Argenes. (D) Euler diagram showing overlap of Nkx3.1-binding sites in WT and HET mouse prostates. See also Supplemental Figure 1 and Supplemental Table 1.

by Nkx3.1. We further used network analysis to identify potential connections between $\mathrm{Nkx} 3.1$ and other transcription factors in target gene regulation. Notably, a link to the protooncogene Myc was identified, suggesting functional interactions between $\mathrm{Nkx} 3.1$ and Myc. We used in vitro and in vivo studies to validate this interaction, showing coregulation of target gene expression and prostate tumorigenicity by Myc and Nkx3.1.

\section{Results}

The Nkx3.1 cistrome in mouse prostate. To define the Nkx3.1 cistrome, we performed ChIP-seq in adult mouse prostate. In the mouse prostate, $\mathrm{Nkx} 3.1$ expression is largely restricted to the luminal epithelial cell compartment $(14,15)$. Thus, interpretation of any signals obtained from Nkx3.1 ChIP-seq analysis of whole prostate tissue is not complicated by potential cell type-specific differences in Nkx3.1-binding patterns. We performed ChIP-seq in $2 \mathrm{Nk} \times 3.1^{+/+}$ (WT), $2 \mathrm{Nk} \times 3.1^{+/-}$(heterozygote [HET]), and, as a biological negative control, $1 \mathrm{Nk} \times 3.1^{-/-}(\mathrm{KO})$ mouse prostates (14). We then used the MACS algorithm (19) to call peaks, which correspond to Nkx3.1-binding sites. Between 13.6 and 15.1 million sequence tags were uniquely mapped to the genome per sample (Supplemental Table 1; supplemental material available online with this article; doi:10.1172/JCI58540DS1).Inclusion of the KO sample as a control showed excellent specificity of the $\alpha-\mathrm{Nkx} 3.1$ antibody used in this assay (Figure 1, A and C). The locations and amplitudes of peaks were strikingly similar between genotypes (WT vs. HET) and biological replicates. Similarity was also seen genome wide (Figure $1 \mathrm{~A}$ ) at the chromosomal level (Figure 1B) and at the level of 

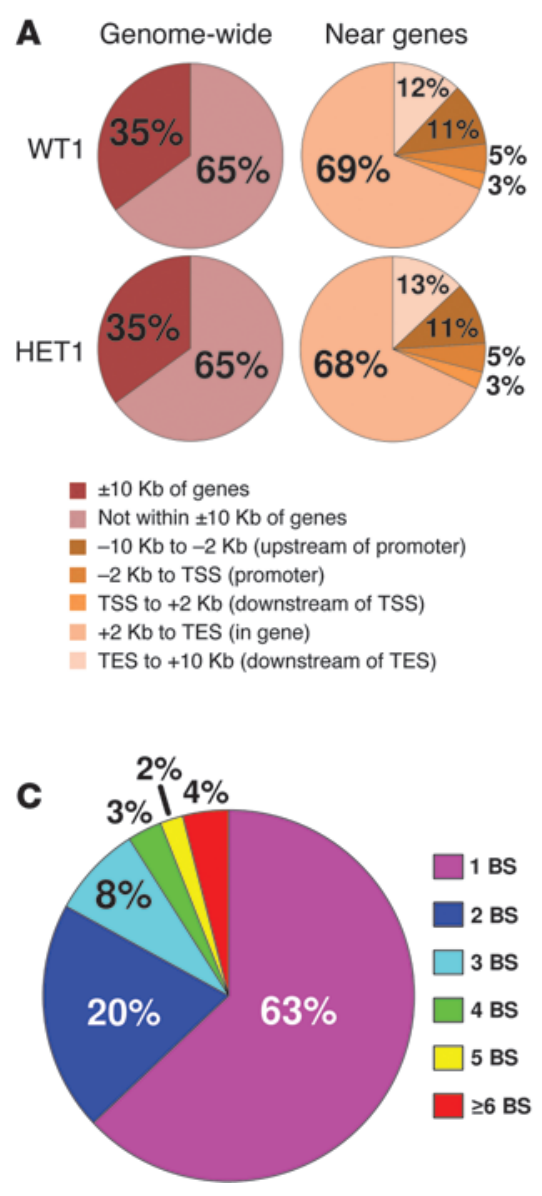

B

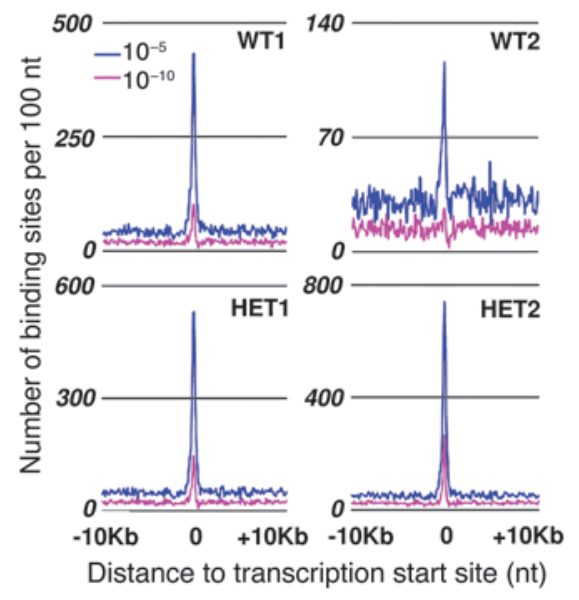

D
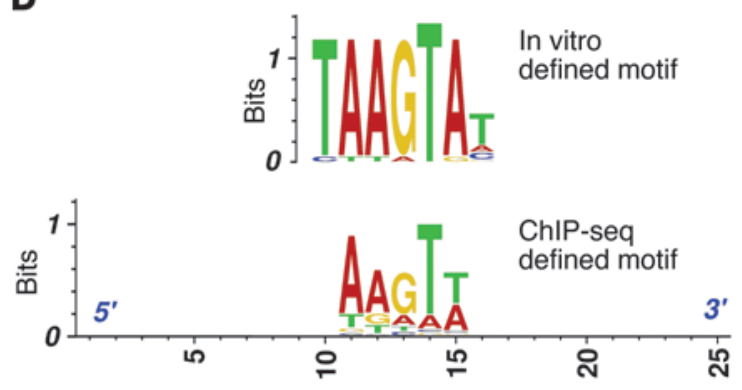

Figure 2

Analysis of the Nkx3.1 cistrome. (A) Spatial distribution of Nkx3.1 binding sites genome wide (red) or near genes as shown in the RefSeqGene database (http://www.ncbi.nlm.nih.gov/refseq/rsg/) (orange). The results from 1 WT prostate and 1 HET prostate are shown. (B) Enrichment of Nkx3.1-binding sites approximately $150 \mathrm{nt}$ upstream of the TSS for all Nkx3.1-positive samples. Shown are results for MACS peak calling algorithm with the $P$ value set at the default $10^{-5}$ and at $10^{-10}$. (C) The number of times Nkx3.1 binds target genes is indicated. bs, binding site. (D) Top panel: in vitro defined Nkx3.1 motif (29). Bottom panel: consensus motif for Nkx3.1, as determined by querying the 1,000 most enriched ChIP-seq loci with MEME (v. 4.6.0). See also Supplemental Tables 2 and 3.

individual gene loci, such as the known Nkx3.1 target genes Pbsn, which encodes Probasin, and $A r$, which encodes androgen receptor (Figure 1C and refs. 18, 20). These results demonstrate a high degree of reproducibility for the assay.

Nkx3.1-binding sites were generally evenly distributed across all chromosomes, with the lowest concentration of binding sites on the Y chromosome, even when the lower number of genes present on the Y chromosome was taken into account (Supplemental Figure 1A). Analysis of all bound genomic loci indicated that 8,298 (34.6\%) were bound in all $4 \mathrm{Nkx} 3.1$-expressing samples (Figure 1D and Supplemental Table 2). Overall, the HET samples had more binding sites than WT samples, which was contrary to our expectations, considering that reduction in $N k \times 3.1$ dosage is highly correlated with prostate tumorigenesis $(5,14,21,22)$. Since ChIP-seq yields a snapshot of cross-linked proteins bound to DNA, it may not be suitable for detecting dosage effects. The majority (65\%) of Nkx3.1-bound intervals mapped within $10 \mathrm{~kb}$ of genes (Figure 2A and Supplemental Table 2), which was consistent with reports showing that other transcription factors prefer to bind in the vicinity of genes (23). The percentage of peaks mapping within 10 $\mathrm{kb}$ of genes was remarkably consistent across all 4 Nkx3.1-positive samples, ranging from 63\% (WT2) to 66\% (HET2) (Figure 2A and Supplemental Table 2). Promoter regions $(-10 \mathrm{~kb}$ to $+2 \mathrm{~kb})$ contained $19 \%$ of all intervals that mapped within $10 \mathrm{~kb}$ of annotated genes. In contrast, $68 \%$ of intervals mapped between $+2 \mathrm{~kb}$ and the transcription end site (TES) (Figure 2A and Supplemental Table 2). These data show a strong preference for $\mathrm{Nkx3} .1$ binding in introns. We discovered a striking enrichment for Nkx3.1-binding sites approximately $-150 \mathrm{nt}$ upstream of the transcription start site (TSS) (Figure 2B). This enrichment was conserved across all 4 Nkx3.1-positive samples and was apparent even at a higher significance threshold $\left(P<10^{-10}\right)$ for peak calling.

Most Nkx3.1 target genes (63\%) were bound once, while $20 \%$ were bound twice, $8 \%$ were bound 3 times, and $9 \%$ were bound 4 or more times (Figure 2C). We next examined for enrichment of known transcription factor DNA-binding motifs in the Nkx3.1bound DNA fragments using Genomatix software (24). We found significant enrichment of several elements, including the NKX homeodomain consensus, which was present in $86 \%$ of sequences examined (Supplemental Table 3). Interestingly, the most enriched 
A
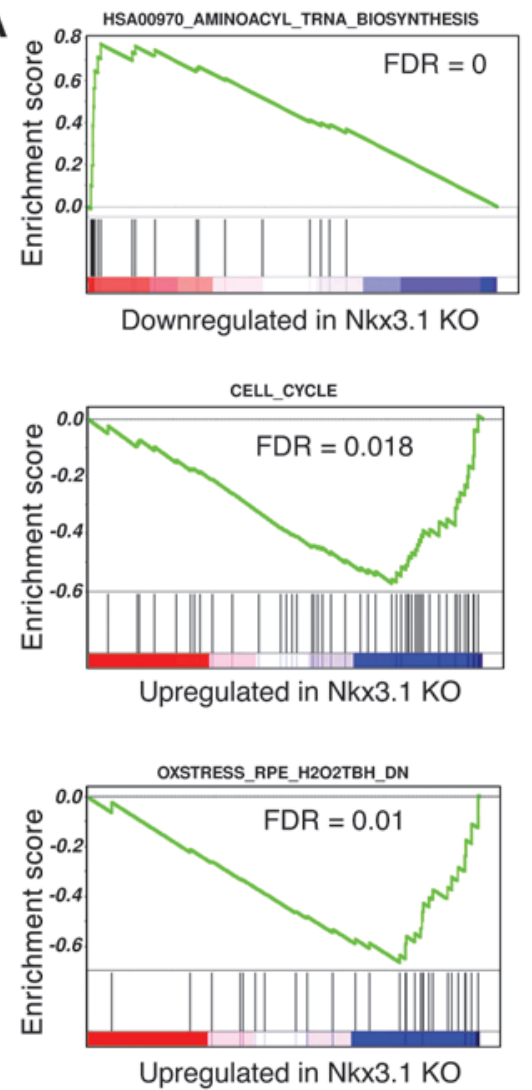

B

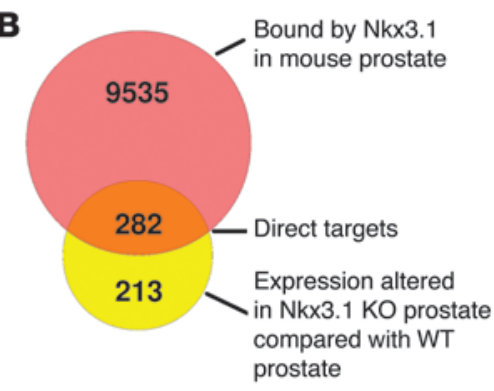

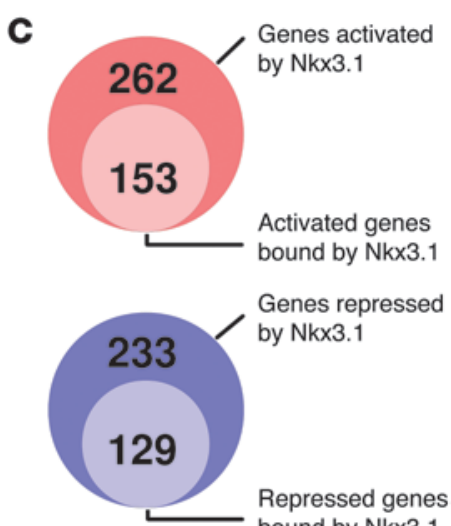

bound by Nkx3.1

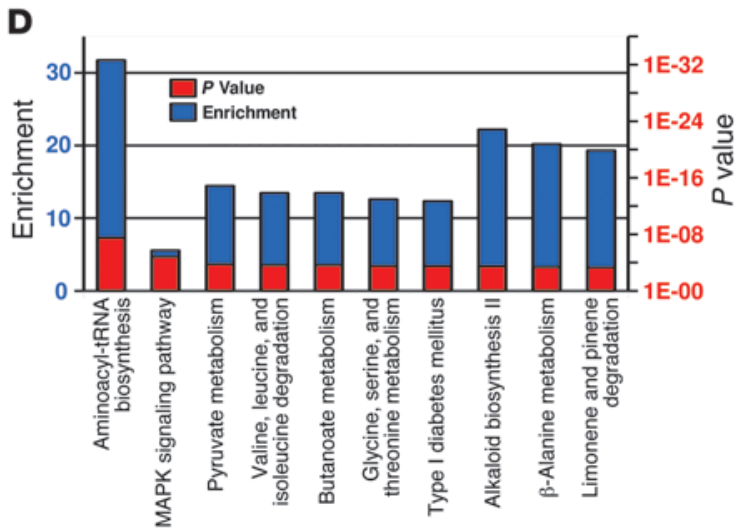

Figure 3

Identification of direct Nkx3.1 target genes. (A) GSEA analysis of genes whose expression is dysregulated in $N k \times 3.1^{-/-}$mice identifies significant downregulation of genes involved in aminoacyl tRNA synthesis and enrichment of genes involved in the cell cycle and oxidative stress in the KO mouse prostate. (B) Integration of Nkx3.1 ChIP-seq data with microarray analysis of Nkx3.1-/- mice identifies 282 direct Nkx3.1 target genes. (C) Venn diagrams showing the fraction of genes activated or repressed by Nkx3.1 that are also bound by Nkx3.1 by ChIP-seq, i.e., direct activated genes and direct repressed genes. (D) GO analysis of direct Nkx3.1 target genes by WebGestalt. See also Supplemental Figure 2 and Supplemental Table 4.

motif, occurring in $94 \%$ of the sequences, was the Forkhead consensus motif. Other enriched motifs included the GATA, Octamer, and TATA-binding factor motifs (Supplemental Table 3). Notably, several of these motifs were reported to be enriched in AR-bound fragments (25-28), although the androgen response element (ARE) was not among the top motifs identified by our analysis (Supplemental Table 3). A putative NKX3.1 DNA-binding motif has been previously defined in vitro using a selection and amplification binding (SAAB) assay (29). Identification of thousands of Nkx3.1-binding sites in our ChIP-seq analysis enabled us to refine the in vivo consensus Nkx3.1 motif. We queried MEME (30) with the 1,000 most enriched regions corresponding to the 1,000 most statistically significant binding sites identified by our ChIP-seq. The motif we discovered, AAGTW (Figure 2D), was similar but not identical to the NKX3.1-binding motif described in vitro, TAAGTA (29). The AAGTW motif was present in approximately $86 \%$ of all intervals (Supplemental Figure 1E).

Identification of direct Nkx3.1 target genes. We next sought to determine "direct" target genes that are both bound and regulated by Nkx3.1. We and others have previously used gene expression profiling to identify genes whose expressions are altered in the prostates of Nkx3.1-deficient mice $(31,32)$. To obtain further insight into gene expression changes in Nkx3.1 KO versus WT prostates, we used Gene Set Enrichment Analysis (GSEA) (33) to reanalyze our microarray data (31). As shown in Figure 3A, we found dramatic changes in gene sets involved in aminoacyl tRNA synthesis, oxidative stress, and notably, cell cycle regulation $(34,35)$ in the $\mathrm{KO}$ prostates. These results reveal a regulatory role for $\mathrm{Nkx} 3.1$ in these processes. Next, we integrated our ChIP-seq data with the microarray data (31). We compared the 9,817 genes bound by Nkx3.1 in the ChIP-seq to the 495 genes changed in the microarray and identified 282 genes present in both lists. These "direct" Nkx3.1 target genes were both bound and regulated by Nkx3.1 (Figure 3B). Based on their expression patterns (i.e., upregulated or downregulated in $\mathrm{KO}$ ), 153 of these genes were inferred to be activated and 129 to be repressed by Nkx3.1 (Figure 3C). Activated Nkx3.1 direct target genes included Tmprss2, Prdx6, Gpx2, and several aminoacyl tRNA synthetase genes (Supplemental Table 4). Repressed direct targets included Angpt2, Hk2, Qsox1, and Nucb2 (Supplemental Table 4). Further analysis indicated that activation or repression by Nkx3.1 does not appear to be related to binding site location within the target genes (Supplemental Figure 2, A and B) or the number of bound sites per gene (P.D. Anderson and S.A. Abdulkadir, unpublished observations). 
A

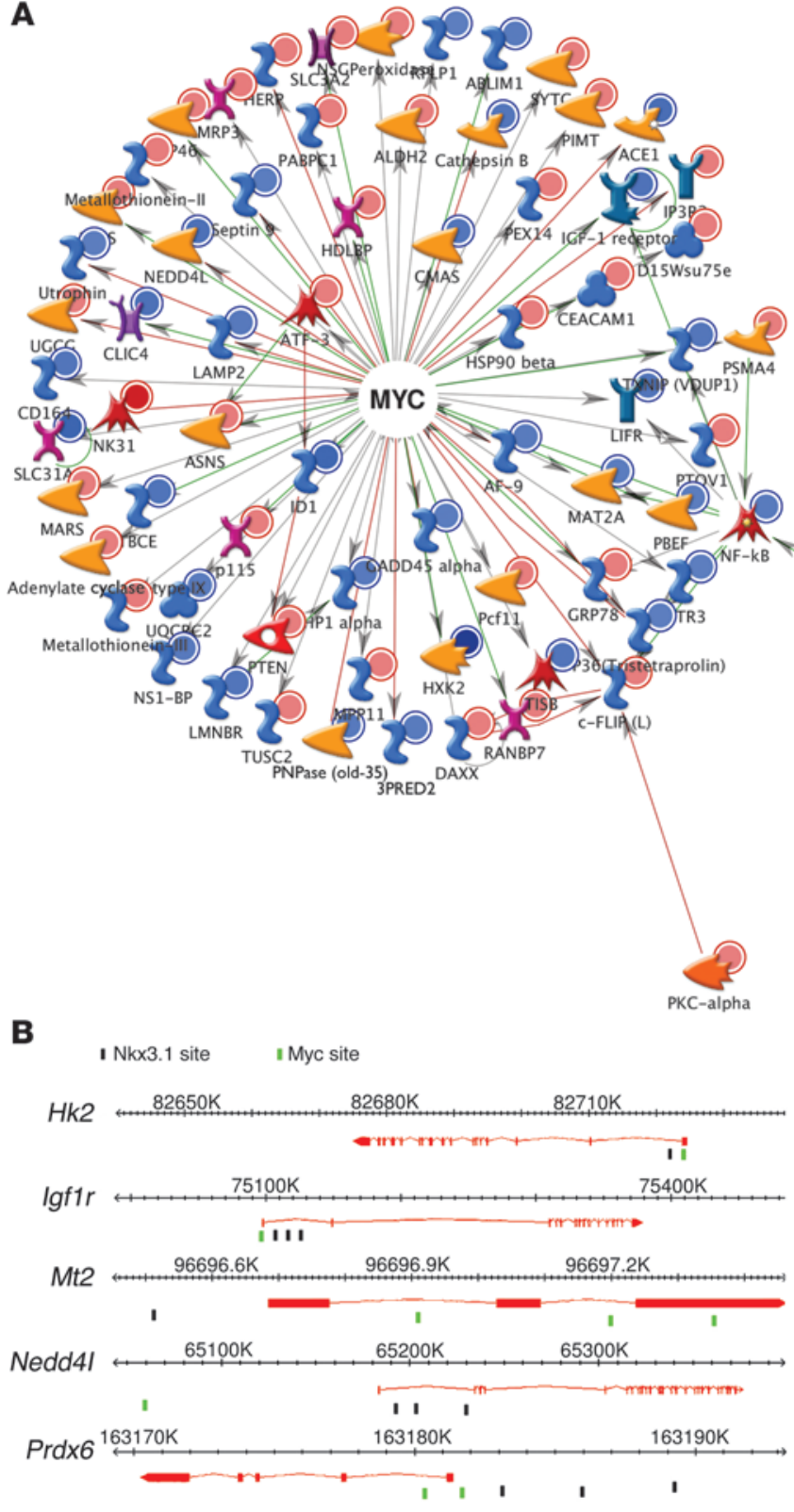

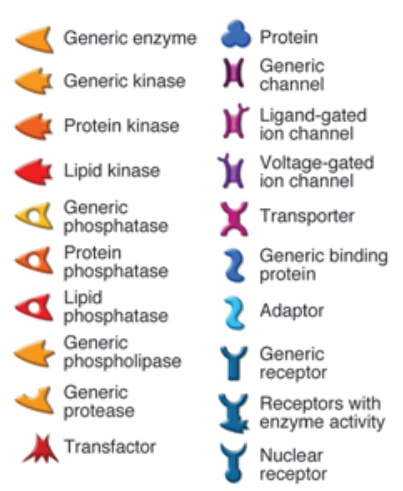

C

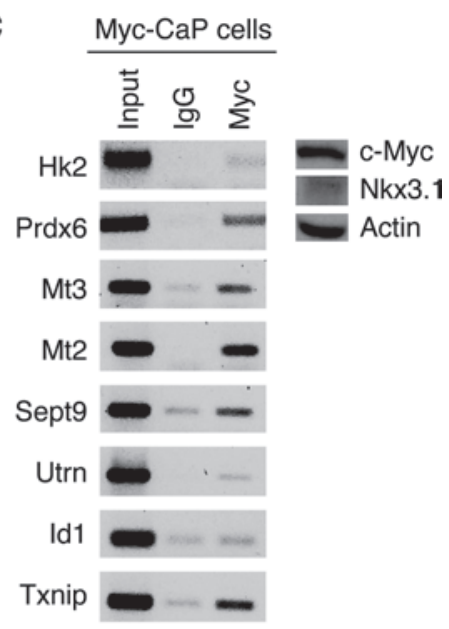

Figure 4

Identification of a subset of direct Nkx3.1 target genes coregulated by Myc. (A) Network analysis using GeneGO MetaCore software identifies a subset of direct Nkx3.1 target genes that are known to be bound by Myc $\left(P=3.94 \times 10^{-169}\right)$. Genes upregulated in the $N k \times 3.1 \mathrm{KO}$ prostates are shown as blue circles, while those downregulated are indicated as red circles in the diagram. (B) Relative locations of actual Nkx3.1- and Mycbinding sites in selected Nkx3.1/Myc coregulated genes identified from genome-wide binding studies. (C) ChIP-PCR validation of Myc binding to selected shared Nkx3.1/Myc target genes in Myc-CaP mouse prostate adenocarcinoma cell line (top). These cells express Myc but not Nkx3.1, as shown in the inset Western blot. Results are representative of at least 2 independent experiments. (D) ChIP-qPCR validation of MYC and NKX3.1 binding to selected shared NKX3.1/MYC target genes in LNCaP human prostate adenocarcinoma cell line. These cells express both MYC and NKX3.1, as shown in the inset Western blot. Results are presented as mean \pm SD from at least 2 independent experiments. See also Supplemental Tables 5 and 6.

We used WebGestalt (36) to test for enrichment of gene ontology (GO) terms among the direct target genes (Figure 3D). Interestingly, genes labeled as components of the aminoacyltRNA synthesis pathway were the most enriched category of GO terms $\left(P<3.0 \times 10^{-8}\right.$, hypergeometric test), which corroborates the GSEA results predicting an important role for $\mathrm{Nkx} 3.1$ in the regulation of protein biosynthesis. Genes in the MAPK signaling pathway were also highly enriched in both the direct targets (Figure 3D) and the ChIP-seq targets overall (Supplemental Figure 1C), suggesting that dysregulation of MAPK pathway members may contribute to the prostate phenotype of Nkx3.1-deficient mice. 
A

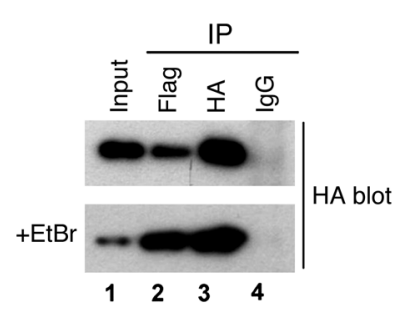

D

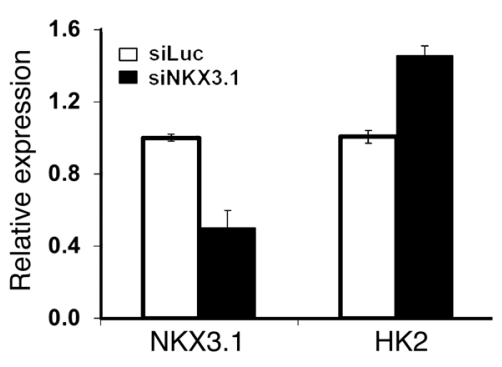

B HA-NKX3.1:

Flag-Myc:

Flag-Myc Mut1:
Flag-Myc Mut2:

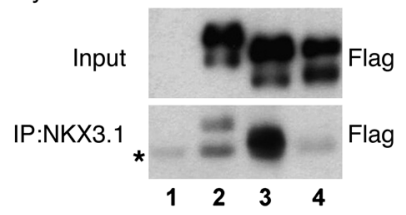

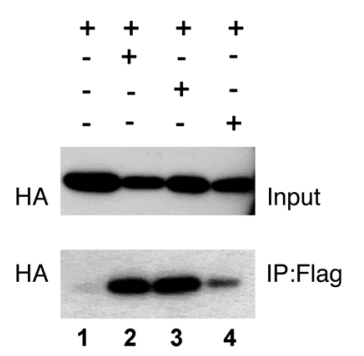

C

HK2

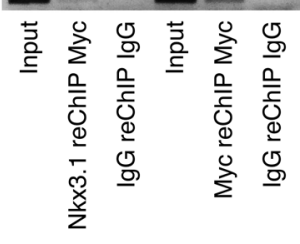

E

Hexokinase 2
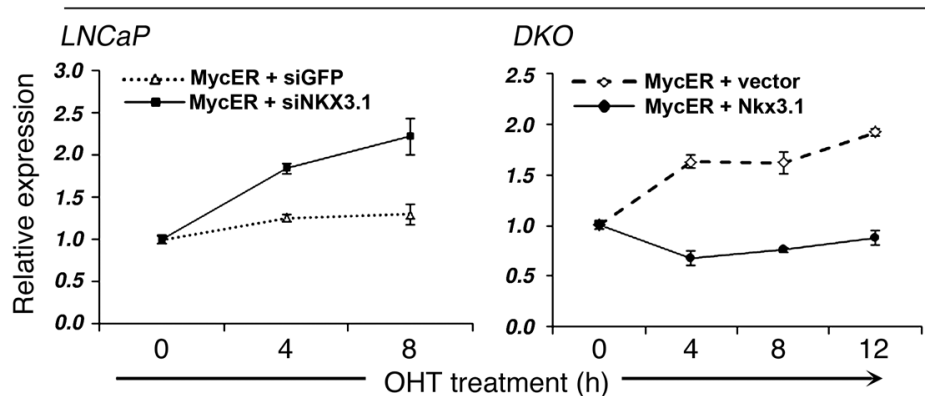

Figure $\mathbf{5}$

NKX3.1 and Myc interact and coregulate expression of shared target gene HK2. (A) Myc and NKX3.1 coimmunoprecipitation. Cell lysates from 293T cells expressing HA-NKX3.1 and Flag-Myc were immunoprecipitated with the indicated antibodies and immunoblotted for HA. To deplete DNA, ethidium bromide (EtBr) was added to lysates for 30 minutes prior to immunoprecipitation and during washes. (B) Coimmunoprecipitation of HA-NKX3.1 and Flag-Myc WT (lane 2), Flag-Myc mutant 1 lacking Myc-box 1 (lane 3), or Flag-Myc mutant 2 lacking Myc-box 2 (lane 4 ) in 293 cells. Asterisk indicates a nonspecific band. (C) ChIP-re-ChIP assay. LNCaP cells were subjected to ChIP by NKX3.1 antibody followed by ChIP with Myc antibody or the reverse and binding interrogated at the HK2 promoter E box. (D) Depletion of NKX3.1 by siRNA in LNCaP cells leads to upregulation of HK2 expression compared with control siLuc (luciferase siRNA) treatment. (E) Depletion of NKX3.1 by siRNA in LNCaPMyc ER cells enhances activation of HK2 gene expression by tamoxifen (OHT) compared with control siGFP treatment (left panel). In contrast, expression of exogenous Nkx3.1 inhibited OHT induction of Hk2 in DKO cells. Results are representative of at least 2 independent experiments. Error bars represent mean \pm SEM. See also Supplemental Figure 4.

Network analysis identifies a gene set coregulated by Nkx3.1 and Myc. To uncover potential relationships between Nkx3.1 target genes, we used the list of direct targets to query the GeneGO MetaCore database (37), which builds gene networks centered on transcription factors. Notably, one of the top ranked networks $\left(P=3.94 \times 10^{-169}\right)$ enriched in direct Nkx3.1 target genes was centered on the protooncogene Myc (Figure 4, A and B, and Supplemental Table 5). In addition, $23 \%$ of the 282 direct $\mathrm{Nkx} 3.1$ targets were also direct Myc targets (Supplemental Table 6). We explored the cellular processes that are regulated by the $65 \mathrm{Nkx} 3.1 / \mathrm{Myc}$ coregulated genes using ClueGO v1.3 (38). ClueGO built a functionally organized network of enriched GO terms using the shared target genes (Supplemental Figure 3). The genes in the established network were enriched in pathways relevant to tumorigenesis, including regulation of telomerase and TNF receptor 2 signaling. These results suggest the intriguing possibility that loss of $N k \times 3.1$ may promote prostate tumorigenesis by dysregulating a set of cancer-related target genes that are also regulated by Myc.

Identification of Myc targets using GeneGO analysis was based on published binding data (usually ChIP-chip or ChIPseq) from various cell lines, such as fibroblasts, lymphocytes, and ES cells. To determine whether Myc may regulate shared target genes identified by GeneGO in the prostate, we used ChIPPCR to validate Myc binding to the shared targets in prostate cancer cells. We used both $\mathrm{Myc}-\mathrm{CaP}$, a mouse prostate epithelial cell line derived from Myc transgenic prostate cancer (39), and LNCaP, a human prostate carcinoma cell line derived from a lymph node metastasis (40). Myc-CaP cells expressed Myc but not Nkx3.1, while LNCaP cells expressed both MYC and NKX3.1 (Figure 4, C and D). We confirmed binding of Myc to 7 of 8 sites tested in Myc-CaP cells, including Hk2, Prdx6, Txnip, Sept9, Mt2, $M t 3$, and Utrn (Figure 4C). In LNCaP cells, we observed binding of MYC and NKX3.1 to their respective sites in 7 genes tested by ChIP-qPCR, including HK2, IGF1R, PRDX6, UTRN, ASNS, CTSB, and $H D L P$ (Figure 4D).

NKX3.1 opposes MYC's transcriptional activity. NKX3.1 is a tumor suppressor, while MYC is an oncoprotein; therefore, we sought to determine whether NKX3.1 can oppose MYC's transcriptional activity on shared target genes. We first determined whether NKX3.1 could physically interact with MYC. Using coimmunoprecipitation analyses, we showed that MYC and NKX3.1 interacted independently of DNA, as depletion of DNA with ethidium bromide did not abrogate the interaction (Figure 5, A and B). Using deletion mutants of MYC, we found that the interaction between MYC and NKX3.1 was severely reduced when MYC box II was deleted (Figure 5B). To determine whether NKX3.1 and MYC can form a complex at the promoter of shared target genes, we performed sequential ChIP in LNCaP cells. Our analysis at the HK2 gene E-box (Figure 5C) demonstrated that NKX3.1 and MYC can form a complex at shared target gene promoters. These results suggest 
A

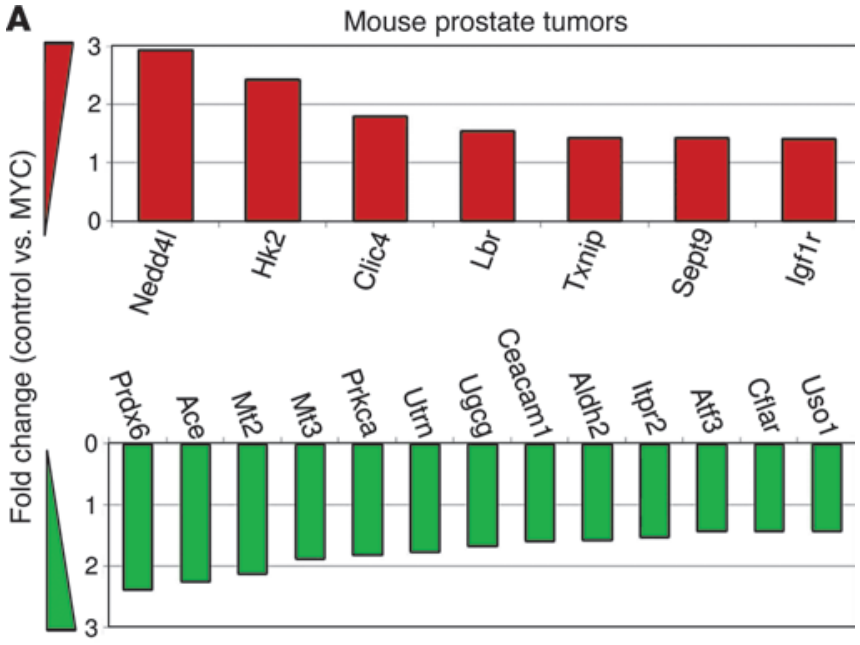

B

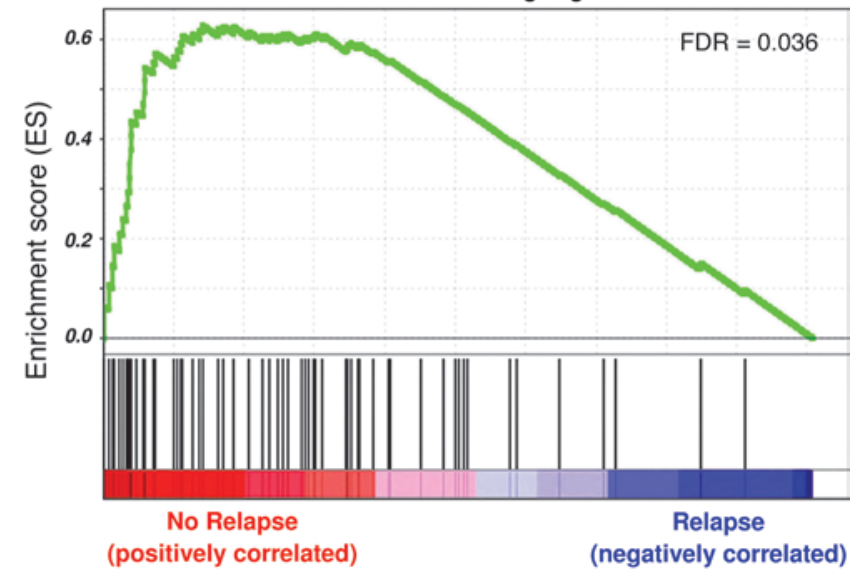

that NKX3.1 may interact with MYC and modulate MYC's transcriptional activity. Support of this was provided by upregulation of HK2 gene expression in LNCaP cells when NKX3.1 was depleted by NKX3.1 siRNA (Figure 5D). Furthermore, in LNCaP cells engineered to express the tamoxifen-inducible MycER protein, depletion of NKX3.1 by siRNA enhanced tamoxifen-induced activation of HK2 gene expression (Figure 5E). Conversely, exogenous Nkx3.1 expression in DKO mouse embryonic fibroblasts (MEFs) expressing MycER impaired the tamoxifen-inducible activation of the Hk2 gene (Figure 5E and Supplemental Figure 4). Overall, these results indicate that NKX3.1 can antagonize MYC's transcriptional activation of shared target genes.

We next sought to determine whether Nkx3.1 and Myc coregulate target gene expression in prostate tissue in vivo. We examined gene expression microarray data obtained from mouse prostate tissues engineered to overexpress MYC with loss of endogenous Nkx3.1. The MYC-overexpressing prostates were generated using tissue recombination, in which naive adult mouse prostate epithelial cells were transduced with control or MYC-expressing lentivirus, combined with fetal rat urogenital mesenchyme (UGM) and grafted under the renal capsule of intact adult male SCID mice (ref. 41 and Supplemental Figure 5A). We have shown previously that these MYC-expressing grafts at 6 weeks contained multiple foci of high-grade prostatic intraepithelial neoplasia (HGPIN),

\section{Figure 6}

Dysregulation of shared Nkx3.1/MYC target genes in mouse and human prostate cancer tissues. (A) Dysregulation of Nkx3.1/MYC targets in a prostate tissue recombination model. Affymetrix arrays were used to compare gene expression between prostate grafts regenerated with mouse prostate epithelial cells transduced with lentivirus overexpressing human MYC or control lentivirus. The MYC grafts contained HGPIN lesions, overexpressed MYC, and downregulated Nkx3.1 (see Supplemental Figure 3). By significance analysis of microarrays (SAM) and employing a 1.4-fold cutoff, 20 of the 65 Nkx3.1/MYC target genes were significantly altered in the grafts containing HGPIN lesions with MYC overexpression and Nkx3.1 downregulation. (B) GSEA analysis shows association between expression of NKX3.1/MYC target genes and relapse in human prostate tumors. Gene expression arrays from tumors consisting of 32 tumors with relapse and 34 tumors without relapse were analyzed. See also Supplemental Figures 3 and 5, and Supplemental Tables 5-7.

while control grafts consisted of benign prostate glands (41). In addition, the HGPIN lesions uniformly lost Nkx3.1 protein expression (Supplemental Figure 5B). We performed microarray analysis on MYC grafts (high MYC/low Nkx3.1) and control grafts (i.e., low MYC/high Nkx3.1) to test for dysregulation in shared target genes (42). At the 1.4-fold cutoff, the expression of 20 of 65 MYC/Nkx3.1 shared targets was significantly altered compared with only 2 of 65 randomly selected genes (false discovery rate $[\mathrm{FDR}]=0.02$; $\left.P=2.9 \times 10^{-5}\right)($ Figure $6 \mathrm{~A})$. Genes significantly upregulated in MYC grafts (i.e., high MYC/low Nkx3.1) included Nedd4l, Hk2, Clic4, and Igf1r, while Prdx6, Ace, Mt2, Prkca, Ugcg, Ceacam1, Itpr2, Cflar, and Atf3 were significantly downregulated (Figure 6A). These results indicate that shared $\mathrm{Nkx} 3.1 / \mathrm{Myc}$ target genes may be relevant to prostate tumorigenesis in vivo.

We next examined microarray data obtained from LNCaP, PC3, and DU145 cells following MYC knockdown by siRNA (43) for evidence of dysregulation of shared NKX3.1/MYC target genes when Myc is depleted. Using a 1.4-fold cutoff, we found significant dysregulation of shared target genes compared with a randomly generated list of genes in all 3 cell lines (Table 1). We also analyzed the concordance in the direction of target gene expression changes (i.e., up or down) between the human cell line siMYC microarray data and the Myc-overexpressing mouse prostate microarray data (Table 1). The concordance rate was high for the PC3 and DU145 cells ( 0.67 and 0.75 , respectively), but lower ( 0.4$)$ for LNCaP cells.

\section{Table 1}

MYC knockdown dysregulates expression of shared NKX3.1/MYC target genes in human prostate cancer cell lines

$\begin{array}{lccc} & \text { LNCaP } & \text { DU145 } & \text { PC3 } \\ \text { Shared NKX3.1/MYC target genes } & 11 & 18 & 24 \\ \text { Random genes } & 4 & 6 & 9 \\ \text { Concordance with mouse TR } & 0.4 & 0.67 & 0.75 \\ \text { Significance: shared over random } & P=0.05 & P=0.006 & P=0.002\end{array}$

Gene expression microarray data for LNCaP, PC3, and DU145 cells treated with siRNA targeting MYC (43) were examined for alterations in NKX3.1/MYC target genes. There was significant alteration in the expression of the shared NKX3.1/MYC targets compared with a randomly generated list of genes. The concordance in the direction of gene expression changes due to MYC modulation in cell lines was determined, as compared with the MYC-expressing mouse tissue microarray data in Figure 6A. TR, tissue recombinants. 
A

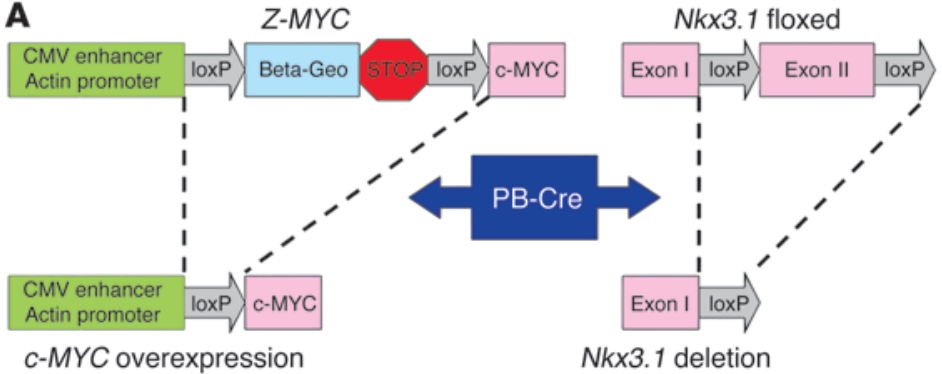

B

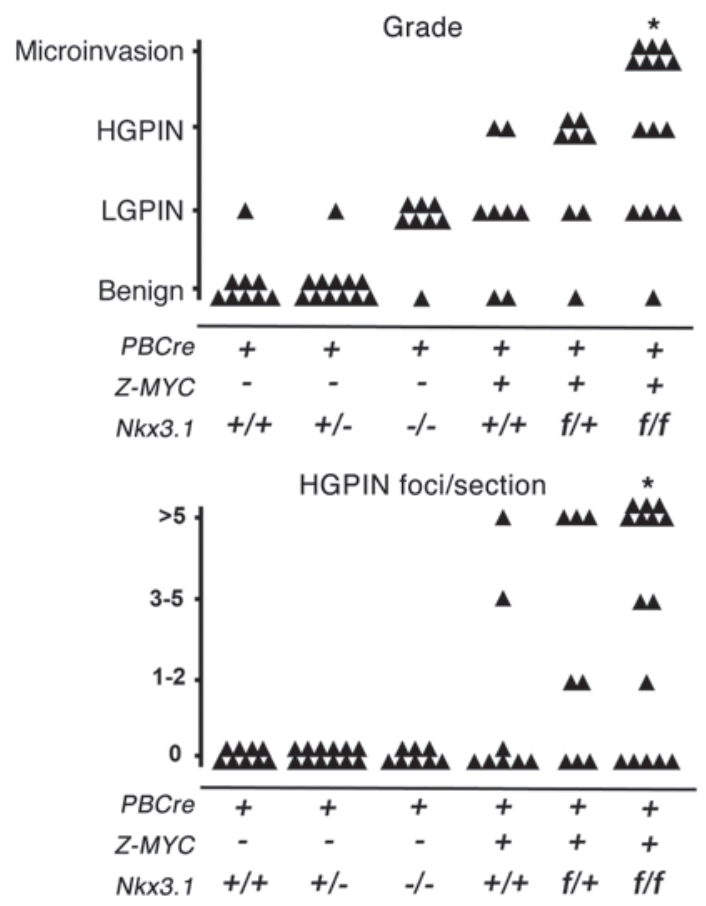

C

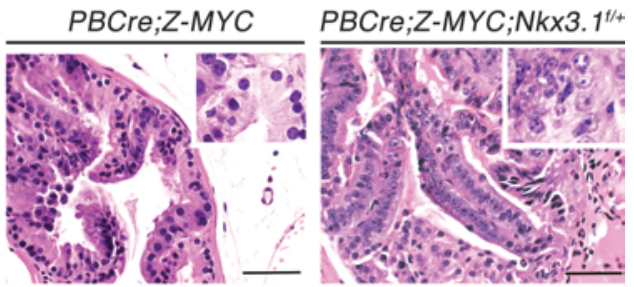

PBCre;Z-MYC;Nkx3. $1^{\text {th }}$

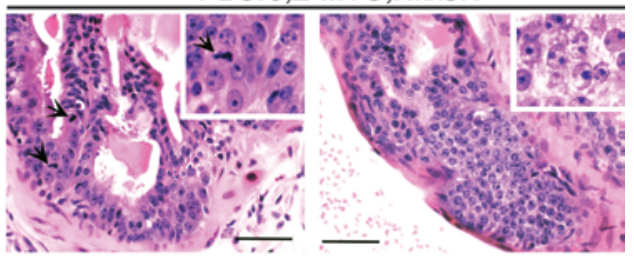

D

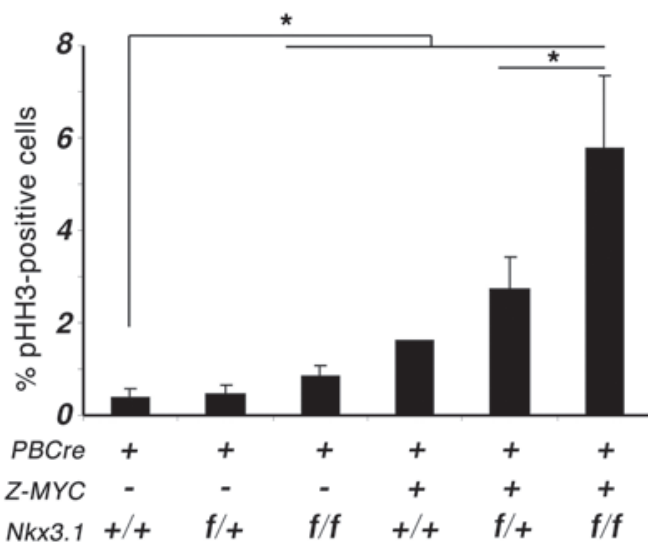

E
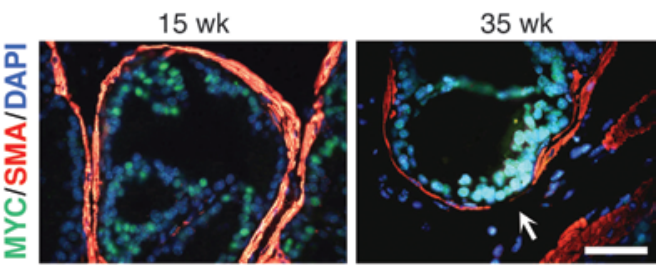

\section{Figure 7}

Loss of Nkx3.1 facilitates MYC-initiated prostate tumorigenesis in transgenic mice. (A) Scheme for concurrent overexpression of MYC and deletion of Nkx3.1 in the prostates of transgenic mice. The Z-MYC construct contains a latent human MYC allele under the control of the CMV enhancer/actin promoter. The floxed allele of $N k x 3.1$ has loxP sites flanking exon 2, which encodes the $N k \times 3.1$ homeodomain. Cre recombinase is under the control of the prostate-specific Probasin promoter $(P B-C r e)$. When Cre is expressed, the MYC gene will be overexpressed and the Nkx3.1 gene will be concurrently deleted. (B) Graph summarizing prostate histology of $N k \times 3.1 / M Y C$ mutant mice. ${ }^{*} P<0.01$ for $P B C r e ; Z-$ MYC;Nkx3.1 $1^{\text {flf }}$ relative to PBCre;Z-MYC. (C) Histological characterization of prostate lesions in Nkx3.1/MYC mutant mice. H\&E-stained sections of 35-week-old mouse prostates. Note high-grade PIN lesions in PBCre;Z-MYC;Nkx3.1 $1^{\text {fl+ }}$ and PBCre;Z-MYC;Nkx3. $1^{\text {flf }}$ mice. Insets show higher magnifications $(\times 40)$. Arrows denote mitotic figures. Scale bars: $50 \mu \mathrm{m}$. (D) Proliferation in mutant mice prostates assessed by phospho-histone H3 staining. ${ }^{*} P<0.05$. Error bars represent mean \pm SEM. (E) Areas of focal loss of SMA (arrows) in 35-week-old PBCre;Z-MYC;Nkx3.1/f mice, suggestive of microinvasion. Scale bar: $50 \mu \mathrm{m}$. See also Supplemental Figures 6 and 7 .

The lower concordance rate in $\mathrm{LNCaP}$ cells may be related to the fact that in these cells, MYC knockdown led to a significant alteration in NKX3.1 levels, thereby confounding the analysis.

We extended our analysis to test for dysregulation of NKX3.1/ MYC coregulated genes in human prostate cancer. We downloaded raw microarray and clinical data for 66 patients from the NCBI Gene Expression Omnibus (GEO GSE21034) (44). The patients were divided into 2 categories based on presence ( 32 patients) or absence (34 patients) of biochemical relapse. Using GSEA, we discovered an association between relapse and expression of MYC/NKX3.1 shared target genes $(\mathrm{FDR}=0.036)$. In tumors from patients without relapse, we found significant upregulation of a subset of shared genes (Figure 6B). The specific genes driving this association were found in the leading edge of the gene set and are listed in Supplemental Table 7. Notably, 9 of the 13 genes $(70 \%)$ downregulated in the Myc-expressing (high Myc/low Nkx3.1) mouse prostate tissues were present in the leading edge of the clinical human array data and upregulated in tumors without 
A

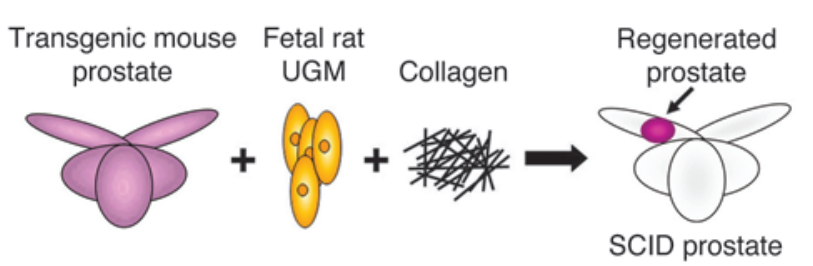

B

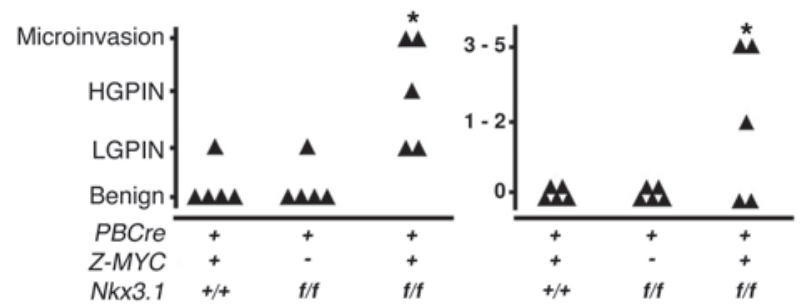

Nkx3.1

$+/+$

f/f
C PBCre;Z-MYC
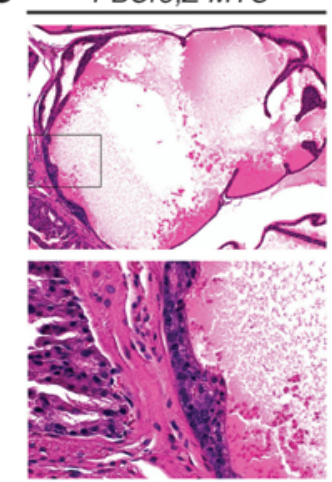

D

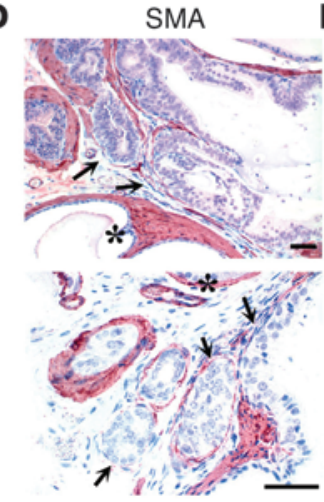

PBCre;Nkx3.1 $1^{\text {ff }}$
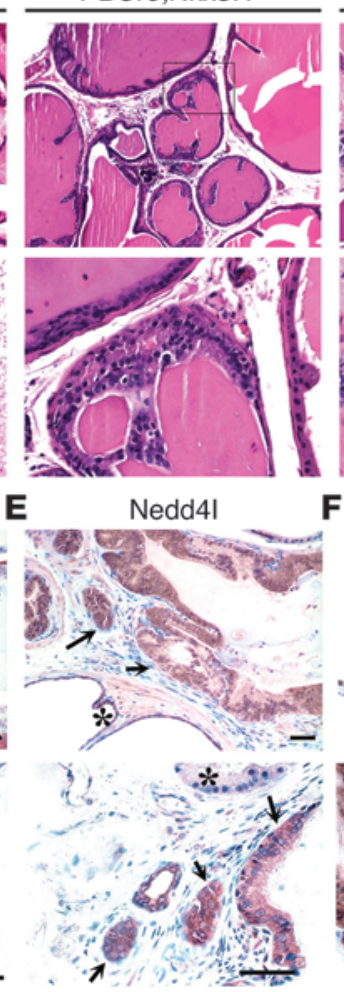

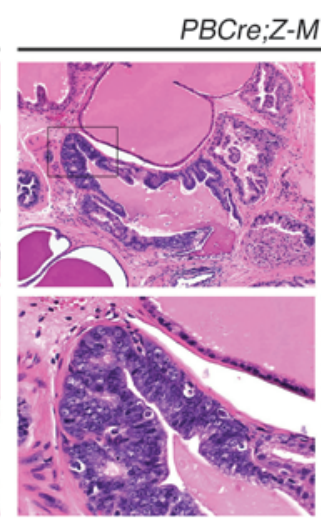

F $\quad H k 2$

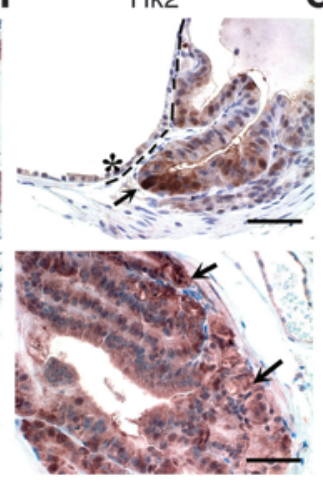

Figure 8

Nkx3.1 and MYC coregulate prostate tumorigenesis and target gene expression. (A) Scheme for prostate regeneration by tissue recombination to recapitulate phenotypes of transgenic mice. Pieces of transgenic mouse prostates were combined with rat UGM and collagen and then implanted orthotopically into prostates of SCID mice. Ten weeks later, regenerated prostates were harvested. (B) Graph summarizing pathology of regenerated prostates. ${ }^{*} P<0.05$ for $P B C r e ; Z-M Y C ; N k \times 3.1^{\text {t/f }}$ relative to all others. (C) Histological characterization of prostate lesions in regenerated prostates. H\&E-stained sections of 10-week-old grafts. PBCre;Z-MYC and PBCre;Nkx3.1/ff grafts show mostly normal glands with areas of hyperplasia and dysplasia consistent with low-grade PIN, while PBCre;Z-MYC;Nkx3.1 f/f prostates show multiple foci of HGPIN. Scale bars: $50 \mu \mathrm{m}$. (D) Focal loss of SMA (arrows) consistent with microinvasion in 10-week-old PBCre;Z-MYC;Nkx3.1/ff prostate grafts. Asterisks indicate benign glands. Scale bars: 50 um. (E-G) Upregulation of Nedd4I and Hk2 and downregulation of Prdx6 expression by immunohistochemistry in HGPIN/cancer lesions from 10-week-old PBCre;Z-MYC;Nkx3.1 $1^{\text {flf }}$ prostate grafts (arrows). Note that the sections in $\mathbf{E}$ are adjacent to those in $\mathbf{D}$. Asterisks indicate benign glands. Scale bars: $50 \mu \mathrm{m}$. See also Supplemental Figures 7 and 8 .

relapse. These genes included UGCG, MT2A, ITPR2, CEACAM1, $A L D H 2, C F L A R, P R K C A$, and ATF3 (Supplemental Table 7). These results suggest that suppression of these shared target genes due to concerted upregulation of MYC and downregulation of NKX3.1 in tumors may favor relapse.

Nkx3.1 loss cooperates with MYC to promote prostate tumorigenesis. Coregulation of target genes by Nkx3.1 and Myc suggests that Nkx3.1 loss and Myc overexpression may cooperate in prostate tumorigenesis. To test this hypothesis in vivo, we generated transgenic mice with concurrent deletion of mouse $N k \times 3.1$ and overexpression of human MYC in the mouse prostate. However, prior to undertaking this in vivo study, we addressed 2 possible conceptual concerns that may complicate the interpretation of this experiment. The first concern was that the promoters commonly used to drive prostate-specific transgene expression, such as Probasin and PSA, are themselves Nkx3.1 target genes. Therefore, Probasin-driven MYC transgene expression, for example, is likely to be decreased in Nkx3.1-deficient prostates. To circumvent this complication, we used Cre/loxP-mediated recombination to concurrently delete endogenous $N k \times 3.1$ and overexpress MYC in the mouse prostate. Specifically, we crossed PbCre4;Z-MYC mice (45, 46) with $N k \times 3.1 / f$ mice (14). Z-MYC is a latent, Cre-activatable MYC 


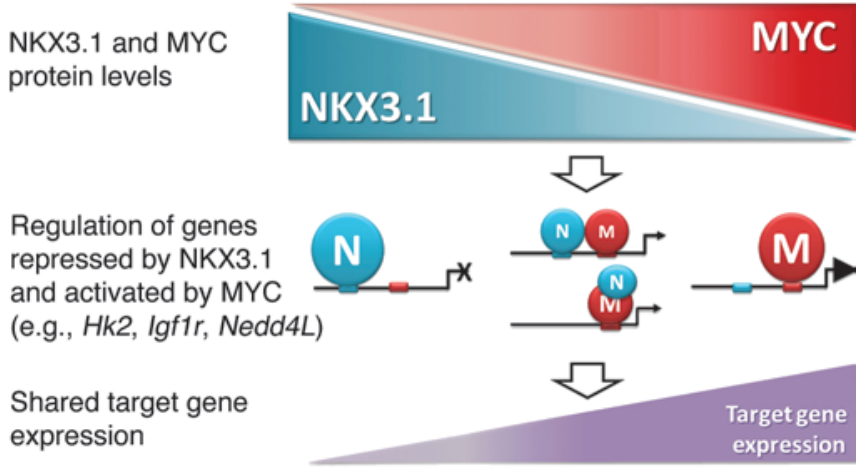

Tumorigenicity as NKX3.1

decreases and MYC increases

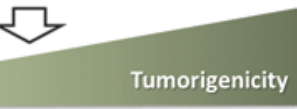

\section{Figure 9}

Model for crossregulation of prostate tumorigenesis by convergence of NKX3.1 and MYC on shared target genes. NKX3.1 and MYC protein levels vary during tumorigenesis, ranging from low MYC/high NKX3.1 in benign tissue to high MYC/low NKX3.1 in more advanced tumors. In benign tissue, where NKX3.1 protein levels are high and MYC levels are very low, shared target genes such as $H K 2$ are bound and repressed by NKX3.1. In samples in which both NKX3.1 and MYC are expressed, NKX3.1 can bind to its consensus DNA site as well as form a complex with MYC to dampen MYC's transcriptional activity and target gene expression. In advanced tumors, where MYC is highly expressed and NKX3.1 expression lost, MYC binds and activates its target genes unopposed. Thus, as tumorigenesis progresses, the MYC:NKX3.1 ratio increases and the expression of protumorigenic shared target genes (activated by MYC and repressed by NKX3.1) increases. In the model depicted here, we show NKX3.1 as a transcriptional repressor and MYC as an activator of protumorigenic target genes. However, the converse, where NKX3.1 activates and MYC represses antitumorigenic target genes, also fits the general model. See also Supplemental Figure 9.

allele. Thus, prostate-specific Cre expression resulted in concurrent deletion of Nkx3.1 and activation of MYC expression (Figure 7A). Because the activated MYC transgene is under the control of the CMV enhancer/actin promoter, loss of $N k \times 3.1$ should not affect transgene expression.

The second concern is that MYC may directly repress Nkx3.1 expression (47), in which case deleting Nkx3.1 would have no added advantage to MYC overexpression in prostate cells. We examined this possibility by double staining PBCre;Z-MYC transgenic mouse prostates for MYC and Nkx3.1. While some MYC-overexpressing cells showed evidence of reduced $\mathrm{Nkx} 3.1$ expression, we observed a number of cells coexpressing MYC and Nkx3.1 (Supplemental Figure 6A). Staining of adjacent serial sections for MYC and Nkx3.1 also showed similar results (Supplemental Figure 6B). Therefore, in this model, MYC overexpression does not immediately lead to downregulation of $\mathrm{Nkx} 3.1$ protein expression. These results are consistent with our previous observations showing that MYC overexpression in PBCre;Z-MYC mice sensitizes cells to transformation, but does not by itself lead to significant pathology, contrary to the case in Probasin-MYC mice (4648). This may be related to differences in background strains or MYC expression levels (47). In sum, our results indicate that the PBCre; $Z$-MYC model is well suited for determining whether Nkx3.1 deletion will modify MYC-induced prostate tumorigenesis in vivo.
We characterized prostate tumorigenesis in cohorts of conditional MYC/Nkx3.1 mutant mice. Prostate-specific deletion of $N k \times 3.1$ using the $P B C r e 4$ line resulted in epithelial hyperplasia and dysplasia (Supplemental Figure 7A), similar to what has been observed using a PSA-Cre line (14). Focal prostate expression of MYC in PbCre4;Z-MYC mice resulted in mild pathology (Figure 7, B and C) as reported earlier (46). Notably, cooperativity between Nkx3.1 loss and MYC overexpression was evident as early as 15 weeks of age in PbCre4;Z-MYC;Nkx3.1f/+ and PbCre4;Z-MYC;Nkx3.1 f/f mice, with $62 \%$ and $67 \%$ developing focal HGPIN lesions, respectively (Figure 7, B and C). Strikingly, 70\% of the PbCre4;Z-MYC;Nkx3.1f/f HGPIN lesions developed microinvasive cancer (Figure 7, B and E). Over time, the density of multifocal HGPIN lesions increased to more than 5 foci per section in PbCre4;Z-MYC;Nkx3.1/f/ mice (Figure 7B). As shown in Figure 7D, proliferation was significantly increased in PbCre4;Z-MYC;Nkx3.1 ${ }^{f /+}$ and PbCre4;Z-MYC;Nkx3.1f/f prostates, while apoptosis showed a modest increase (S.A. McKissic and S.A. Abdulkadir, unpublished observations).

We extended these results by prostate regeneration using tissue recombination. Prostates regenerated by tissue recombination recapitulate the prostate phenotypes of transgenic mice (49), with the added benefits of savings in time and cost. In this assay (Figure 8A), we combined pieces of adult transgenic mouse prostate tissue with fetal UGM. Tissue recombinants were then grafted orthotopically into the anterior prostates of SCID mice. Then, 6 or 10 weeks later, grafts containing regenerated prostates were harvested. Regenerated glands can be distinguished from host SCID mouse prostate glands by MYC expression and/or loss of Nkx3.1 expression (Supplemental Figure 7C). Histological examination of grafts showed that while PbCre4;Z-MYC and PbCre4;Nkx3.1f/f grafts were either histologically normal or developed epithelial hyperplasia and LGPIN, grafts from PbCre4;Z-MYC;Nkx3.1f/f mice contained HGPIN lesions with microinvasive cancer (Figure 8, B-D, and Supplemental Figure 7D). We next sought to determine whether the expression of $\mathrm{Nkx3.1/MYC}$ coregulated genes was altered in Nkx3.1/MYC mutant mice. Using immunohistochemistry, we examined the expression of Hexokinase 2 ( $\mathrm{Hk} 2)$, a key glycolic isoenzyme, Nedd4l, an E3 ubiquitin ligase, and Prdx6, an antioxidant enzyme, in prostate tissue sections from PbCre4;Z-MYC;Nkx3.1f/f mouse grafts. We observed significant upregulation of $\mathrm{Hk} 2$ and Nedd4l and downregulation of Prdx6 proteins in the HGPIN/ microinvasive carcinoma lesions (Figure 8, E-G, and Supplemental Figure 8). These results support a role for these coregulated target genes in prostate tumorigenesis.

\section{Discussion}

In this work, we found significant overlap between targets of the prostate tumor suppressor Nkx3.1 and Myc using integrated genomic analysis. Prior to this study, few direct targets of the Nkx3.1 tumor suppressor were known. By integrating ChIP-seq and gene expression data, we defined a set of 282 direct target genes both bound and regulated by Nkx3.1. This number probably represents an underestimate, given the use of older platforms in the 2 microarray studies that catalogued genes with dysregulated expressions in the prostates of $N k \times 3.1$ mice $(31,32)$.

It is notable that Nkx3.1 binding is enriched near genes, particularly around $150 \mathrm{nt}$ upstream of the TSS. Nkx3.1 has been implicated in regulating the production of secretions in the differentiated prostate, and we found that Nkx3.1-bound genes were enriched in processes relevant to this function, including amino 
acyl-tRNA synthesis, amino acid metabolism, and regulation of oxidative stress. The availability of the Nkx3.1 cistrome enabled us to refine the in vivo consensus Nkx3.1-binding motif from TAAGTA to AAGTW and provides the scientific community with a comprehensive resource for further investigation of Nkx3.1 function in specific cell types. For example, Nkx3.1 has been implicated in regulating self renewal of specific types of prostate stem cells called castration-resistant Nkx3.1-expressing cells (CARNs) and in regulating $\mathrm{T}$ cell lymphomagenesis $(6,50)$. In these settings, Nkx3.1 may regulate a distinct set of genes compared with those it regulates in differentiated prostate epithelium.

It is striking that many of the direct Nkx3.1 targets are also targets of the Myc oncoprotein. Several of these "shared" Nkx3.1/ Myc targets have been implicated in various aspects of tumorigenesis. For example, Hk2, a key glycolytic enzyme, is involved in promoting the Warburg effect and tumorigenesis in cancer cells (51). The E3 ubiquitin ligase Nedd41, which is involved in targeting components of the TGF- $\beta$ pathway for degradation, was found in some studies to be upregulated in prostate cancer (52). It is likely that dysregulation of multiple Nkx3.1/Myc target genes contributes to malignant transformation. Myc is a wellknown regulator of proliferation, differentiation, and tumorigenesis (53). Thus, some of the consequences of Nkx3.1 loss in the prostate, including hyperproliferation and dedifferentiation $(5,14)$, may be achieved via direct dysregulation of the shared targets. In human prostate cancer, overexpression of MYC is an early and frequent event $(41,54)$. The expression of NKX3.1 is also often reduced in human prostate tumors $(22,55)$, and an inherited variant of the NKX3.1 gene with reduced expression is associated with increased risk of prostate cancer (12).

Based on our studies, we propose the following model to explain the relationship between NKX3.1 and MYC in prostate tumorigenesis (Figure 9). The levels of NKX3.1 and MYC vary in tumorigenesis, with the MYC:NKX3.1 ratio increasing with progression. Our data suggest that NKX3.1 and MYC directly bind and coregulate a common subset of target genes relevant to prostate tumorigenesis (Supplemental Figure 9). We propose that NKX3.1 opposes MYC's transcriptional activity. Thus, as the MYC:NKX3.1 ratio increases, MYC's transactivation of protumorigenic target genes (such as HK2, IGF1R, NEDD4L) increases, thereby promoting tumorigenesis. In the case of antitumorigenic target genes (e.g., ACE, MT2) that are repressed by MYC and activated by NKX3.1, the converse is true. As the MYC:NKX3.1 ratio increases, the expression of these target genes will decrease, enhancing tumorigenesis.

Although our study focused on how Nkx3.1 modulates Myc function, Myc may also modulate Nkx3.1 function. Nkx3.1 and Myc may modulate each other's transcriptional activity in several ways. For example, Nkx3.1 and Myc may affect each other's recruitment to chromatin. Alternatively, binding of $\mathrm{Nkx} 3.1$ to promoters/enhancers may affect Myc's transactivation function by interfering with Myc's ability to assemble an active transcription initiation complex. The converse may also be true for target genes that are repressed by Myc. Additional studies are needed to examine these possibilities. Myc's transactivation function may be modulated by complex formation with other factors on chromatin. For example, p19ARF could associate with Myc on chromatin, opposing the transactivation of selected Myc target genes $(56,57)$. Myc can also be recruited to promoter regions via binding to the transcription factor MIZ-1 to repress expression of some target genes $(58,59)$. Finally, although not all shared target genes are coordinately regulated by Myc and Nkx3.1, our analysis provides evidence for cooperation between Myc overexpression and Nkx3.1 loss in promoting cancer and dysregulating multiple shared target genes known to be involved in tumorigenesis. The type of interaction we identified between MYC and Nkx3.1, whereby 2 transcription factors converge to crossregulate common target genes, may provide a mechanistic basis for some oncogene/TSG cooperativity.

\section{Methods}

Mice. Nkx3.1 $1^{-1-}, N k \times 3.1^{f / f}$, and PBcre4;Z-MYC mice have been described (14, $45,46)$. Alleles were maintained on a recombinant inbred BL6/129 background. Nkx3.1 $1^{+/-}$mice were brother-sister-mated to produce the males used in this study.

ChIP-seq assays. Whole prostates from $N k \times 3.1^{+/+}, N k \times 3.1^{+/-}$, and $N k \times 3.1^{-/-}$ mice at 12 to 16 weeks of age were used. Initial ChIP using anti-Nkx3.1 antibody (sc-15022; Santa Cruz Biotechnology Inc.) was performed by Genpathway. HudsonAlpha performed deep sequencing. The genome coordinates of each read were inferred based on sequence homology to mouse genome build 37. Peaks were called using the model-based analysis of ChIPseq (MACS) algorithm (19) using the KO sample as a control for nonspecific Nkx3.1 antibody binding. Further details on ChIP assay can be found in the Supplemental Methods.

Binding site motif analysis. We used Genomatix MatInspector (24) to search 1,000 randomly selected Nkx3.1 intervals for known transcription factorbinding sites. In addition, we performed de novo motif discovery using MEME 4.6.0 (30). The MEME settings were as follows: 1 motif per sequence (OOPS model), minimum motif width $=6$, maximum motif width $=20$. To search for the AAGTW Nkx3.1 motif in Nkx3.1-bound intervals, peaks were divided into 10 quantiles based on their amplitude. Q1 had the tallest peaks and Q10 the shortest. FIMO (part of MEME suite 4.6.0) was used to search significant ChIP-seq intervals for the consensus Nkx3.1 motif that was discovered using MEME. All sequences were screened using RepeatMasker (version 3.2.9) before analysis using the mouse repeat libraries (RepBase 2009-06-04; Genetic Information Research Institute).

Network analysis. The 282 direct Nkx3.1 target genes were uploaded into the MetaCore analytical suite (GeneGO) and analyzed as described previously (37). We built networks centered on transcription factors such that subnetworks centered on transcription factors were generated, with subnetworks ranked by a $P$ value and a G score. Subnetworks were also interpreted in terms of GO.

GSEA. For Nkx3.1 mutant mice, the curated gene sets of the Molecular Signatures Database (MSigDB version 2.5) were tested for enrichment in robust multichip averaging-normalized (RMA-normalized) microarray expression data from a previously published microarray study (31). For human tumors, we obtained raw gene expression microarray data with matching clinical data for 66 human prostate adenocarcinoma patients from Gene Expression Omnibus (GEO GSE21034) (44). The microarray data were processed and converted to RMA-normalized expression values in Bioconductor using XPS. The patients were divided into 2 populations based on presence (32 patients) or absence (34 patients) of biochemical relapse within 5 years. GSEA v2.07 (33) was used to test for expression changes in a gene set consisting of 65 NKX3.1/MYC coregulated genes.

Prostate regeneration by tissue recombination. This was performed generally as described before (41). Briefly, 8- to 10-week-old PBCre;Z-MYC, PBCre;Nkx3.1fff, or PBCre;Z-MYC;Nkx3.1 f/f mouse prostate tissues were minced, combined with freshly isolated fetal rat UGM cells, and incubated in collagen overnight. The next day, tissue recombinants were grafted under the capsule of 1 anterior prostate lobe of 7- to 8-week-old SCID mice and, 6 or 10 weeks later, grafts were harvested. 
Statistics. We employed the hypergeometric test, Fisher's exact test, and the 2-tailed Student's $t$ test for statistical analysis. $P \leq 0.05$ was considered significant. Unless indicated otherwise, error bars represent mean \pm SEM.

Study approval. All procedures were approved by the Vanderbilt University Institutional Animal Care and Use Committee (IACUC).

Accession numbers. The short-read sequencing data have been deposited in the National Center for Biotechnology Short Read Archive (GEO GSE35971).

\section{Acknowledgments}

We thank Pia Arrate and Erin Martinez for technical assistance. This work was supported by grants CA094858 and CA123484 from the National Cancer Institute (NCI) and W81XWH-09-10439 from the Department of Defense (DoD) (to S.A. Abdulkadir). C.M. Eischen was supported by CA148950, and S.W. Hayward was supported by U54 CA126505 from the NCI. P.D. Anderson was supported by a DoD postdoctoral fellowship award (W81XWH-111-0230). S.A. McKissic was supported by award 3R01CA09485807S1 from the National Cancer Institute. The content is solely the responsibility of the authors and does not necessarily represent the official views of the National Cancer Institute or the NIH.

Received for publication April 15, 2011, and accepted in revised form February 22, 2012.

Address correspondence to: Sarki Abdulkadir, Department of Pathology, Microbiology and Immunology, 116121 st Ave. South, B-MCN-3321A, Nashville, Tennessee 37232, USA. Phone: 615.322.9668; Fax: 615.322.7023; E-mail: sarki.abdulkadir@ vanderbilt.edu.
1. Land H, Parada LF, Weinberg RA. Tumorigenic conversion of primary embryo fibroblasts requires at least two cooperating oncogenes. Nature. 1983;304(5927):596-602.

2. Ruley HE. Adenovirus early region $1 \mathrm{~A}$ enables viral and cellular transforming genes to transform primary cells in culture. Nature. 1983;304(5927):602-606.

3. McMurray HR, et al. Synergistic response to oncogenic mutations defines gene class critical to cancer phenotype. Nature. 2008;453(7198):1112-1116.

4. Luo J, Elledge SJ. Cancer: Deconstructing oncogenesis. Nature. 2008;453(7198):995-996.

5. Bhatia-Gaur R, et al. Roles for Nkx3.1 in prostate development and cancer. Genes Dev. 1999; 13(8):966-977.

6. Wang $X$, et al. A luminal epithelial stem cell that is a cell of origin for prostate cancer. Nature. 2009;461(7263):495-500.

7. Abdulkadir SA. Mechanisms of prostate tumorigenesis: roles for transcription factors $\mathrm{Nkx3.1}$ and Egr1. Ann N Y Acad Sci. 2005;1059:33-40.

8. Abate-Shen C, Shen MM, Gelmann E. Integrating differentiation and cancer: the Nkx3.1 homeobox gene in prostate organogenesis and carcinogenesis. Differentiation. 2008;76(6):717-727.

9. Zheng SL, et al. Germ-line mutation of NKX3.1 cosegregates with hereditary prostate cancer and alters the homeodomain structure and function. Cancer Res. 2006;66(1):69-77.

10. Eeles RA, et al. Identification of seven new prostate cancer susceptibility loci through a genome-wide association study. Nat Genet. 2009;41(10):1116-1121.

11. Takata R, et al. Genome-wide association study identifies five new susceptibility loci for prostate cancer in the Japanese population. Nat Genet. 2010;42(9):751-754.

12. Akamatsu S, et al. A functional variant in NKX3.1 associated with prostate cancer susceptibility down-regulates NKX3.1 expression. Hum Mol Genet. 2010;19(21):4265-4272.

13. Kim MJ, et al. Nkx3.1 mutant mice recapitulate early stages of prostate carcinogenesis. Cancer Res. 2002;62(11):2999-3004.

14. Abdulkadir SA, et al. Conditional loss of nkx3.1 in adult mice induces prostatic intraepithelial neoplasia. Mol Cell Biol. 2002;22(5):1495-1503.

15. Kim MJ, et al. Cooperativity of Nkx3.1 and Pten loss of function in a mouse model of prostate carcinogenesis. Proc Natl Acad Sci U S A. 2002;99(5):2884-2889.

16. Abate-Shen C, et al. Nkx3.1; Pten mutant mice develop invasive prostate adenocarcinoma and lymph node metastases. Cancer Res. 2003; 63(14):3886-3890.

17. Wang S, et al. Prostate-specific deletion of the murine Pten tumor suppressor gene leads to metastatic prostate cancer. Cancer Cell. 2003;4(3):209-221.

18. Lei Q, et al. NKX3.1 stabilizes p53, inhibits AKT activation, and blocks prostate cancer initiation caused by PTEN loss. Cancer Cell. 2006;9(5):367-378.

19. Zhang Y, et al. Model-based analysis of ChIP-Seq (MACS). Genome Biol. 2008;9(9):R137.

20. Mogal AP, van der Meer R, Crooke PS, Abdulkadir SA. Haploinsufficient prostate tumor suppression by $\mathrm{Nkx3}$.1: a role for chromatin accessibility in dosage-sensitive gene regulation. J Biol Chem. 2007;282(35):25790-25800.

21. He WW, et al. A novel human prostate-specific, androgen-regulated homeobox gene (NKX3.1) that maps to $8 \mathrm{p} 21$, a region frequently deleted in prostate cancer. Genomics. 1997;43(1):69-77.

22. Bowen C, et al. Loss of NKX3.1 expression in human prostate cancers correlates with tumor progression. Cancer Res. 2000;60(21):6111-6115.

23. O'Geen H, et al. Genome-wide binding of the orphan nuclear receptor TR4 suggests its general role in fundamental biological processes. BMC Genomics. 2010;11:689.

24. Cartharius K, et al. MatInspector and beyond: promoter analysis based on transcription factor binding sites. Bioinformatics. 2005;21(13):2933-2942.

25. Wang $Q$, et al. A hierarchical network of transcription factors governs androgen receptor-dependent prostate cancer growth. Mol Cell. 2007;27(3):380-392.

26. Lupien $M$, et al. FoxA1 translates epigenetic signatures into enhancer-driven lineage-specific transcription. Cell. 2008;132(6):958-970.

27. $\mathrm{He} \mathrm{HH}$, et al. Nucleosome dynamics define transcriptional enhancers. Nat Genet. 2010;42(4):343-347.

28. Yu J, et al. An integrated network of androgen receptor, polycomb, and TMPRSS2-ERG gene fusions in prostate cancer progression. Cancer Cell. 2010;17(5):443-454.

29. Steadman DJ, Giuffrida D, Gelmann EP. DNAbinding sequence of the human prostate-specific homeodomain protein NKX3.1. Nucleic Acids Res. 2000;28(12):2389-2395

30. Bailey TL, Elkan C. Fitting a mixture model by expectation maximization to discover motifs in biopolymers. Proc Int Conf Intell Syst Mol Biol. 1994;2:28-36.

31. Magee JA, Abdulkadir SA, Milbrandt J. Haploinsufficiency at the Nkx3.1 locus. A paradigm for stochastic, dosage-sensitive gene regulation during tumor initiation. Cancer Cell. 2003;3(3):273-283.

32. Ouyang X, DeWeese TL, Nelson WG, Abate-Shen C. Loss-of-function of Nkx3.1 promotes increased oxidative damage in prostate carcinogenesis. Cancer Res. 2005;65(15):6773-6779.

33. Subramanian A, et al. Gene set enrichment analysis: a knowledge-based approach for interpreting genome-wide expression profiles. Proc Natl Acad Sci US A. 2005;102(43):15545-15550.

34. Kanehisa M, Goto S. KEGG: kyoto encyclopedia of genes and genomes. Nucleic Acids Res. 2000; 28(1):27-30.

35. Weigel AL, Handa JT, Hjelmeland LM. Microarray analysis of H2O2-, HNE-, or tBH-treated ARPE-19 cells. Free Radic Biol Med. 2002;33(10):1419-1432.

36. Zhang B, Kirov S, Snoddy J. WebGestalt: an integrated system for exploring gene sets in various biological contexts. Nucleic Acids Res. 2005;33(web server issue):W741-W748.

37. Nikolsky Y, Nikolskaya T, Bugrim A. Biological networks and analysis of experimental data in drug discovery. Drug Discov Today. 2005;10(9):653-662.

38. Bindea G, et al. ClueGO: a Cytoscape plug-in to decipher functionally grouped gene ontology and pathway annotation networks. Bioinformatics. 2009;25(8):1091-1093.

39. Watson PA, Ellwood-Yen K, King JC, Wongvipat J, Lebeau MM, Sawyers CL. Context-dependent hormone-refractory progression revealed through characterization of a novel murine prostate cancer cell line. Cancer Res. 2005; 65(24):11565-11571.

40. Horoszewicz JS, et al. LNCaP model of human prostatic carcinoma. Cancer Res. 1983;43(4):1809-1818.

41. Wang J, et al. Pim 1 kinase synergizes with c-MYC to induce advanced prostate carcinoma. Oncogene. 2010;29(17):2477-2487.

42. Wang J, Anderson PD, Luo W, Gius D, Roh M, Abdulkadir SA. Pim 1 kinase is required to maintain tumorigenicity in MYC-expressing prostate cancer cells [published online ahead of print August 22, 2011]. Oncogene. doi:10.1038/onc.2011.371.

43. Koh CM, et al. Alterations in nucleolar structure and gene expression programs in prostatic neoplasia are driven by the MYC oncogene. Am J Pathol. 2011;178(4):1824-1834.

44. Taylor BS, et al. Integrative genomic profiling of human prostate cancer. Cancer Cell. 2010; 18(1):11-22.

45. Roh M, Kim J, Song C, Wills M, Abdulkadir SA. Transgenic mice for Cre-inducible overexpression of the oncogenes c-MYC and Pim-1 in multiple tissues. Genesis. 2006;44(10):447-453.

46. Kim J, Eltoum IE, Roh M, Wang J, Abdulkadir SA. Interactions between cells with distinct mutations in c-MYC and Pten in prostate cancer. PLoS Genet. 2009;5(7):e1000542.

47. Iwata $T$, et al. MYC overexpression induces prostatic intraepithelial neoplasia and loss of $\mathrm{Nkx} 3.1$ in mouse luminal epithelial cells. PLoS One. 2010;5(2):e9427.

48. Ellwood-Yen K, et al. Myc-driven murine prostate cancer shares molecular features with human prostate tumors. Cancer Cell. 2003;4(3):223-238.

49. Ishii K, Shappell SB, Matusik RJ, Hayward SW. Use of tissue recombination to predict phenotypes of transgenic mouse models of prostate carcinoma. Lab Invest. 2005;85(9):1086-1103.

50. Kusy S, et al. NKX3.1 is a direct TAL1 target gene that mediates proliferation of TAL1-expressing human $\mathrm{T}$ cell acute lymphoblastic leukemia. J Exp Med. 2010;207(10):2141-2156. 
51. Mathupala SP, Ko YH, Pedersen PL. Hexokinase-2 bound to mitochondria: cancer's stygian link to the "Warburg Effect" and a pivotal target for effective therapy. Semin Cancer Biol. 2009;19(1):17-24.

52. Hellwinkel OJ, et al. Transcription alterations of members of the ubiquitin-proteasome network in prostate carcinoma. Prostate Cancer Prostatic Dis. 2011;14(1):38-45.

53. Dang CV, O’Donnell KA, Zeller KI, Nguyen T, Osthus RC, Li F. The c-Myc target gene network. Semin Cancer Biol. 2006;16(4):253-264.
54. Gurel B, et al. Nuclear MYC protein overexpression is an early alteration in human prostate carcinogenesis. Mod Pathol. 2008;21(9):1156-1167.

55. Bethel CR, et al. Decreased NKX3.1 protein expression in focal prostatic atrophy, prostatic intraepithelial neoplasia, and adenocarcinoma: association with gleason score and chromosome 8 p deletion. Cancer Res. 2006;66(22):10683-10690.

56. Qi Y, Gregory MA, Li Z, Brousal JP, West K, Hann SR. p19ARF directly and differentially controls the functions of c-Myc independently of p53. Nature.
2004;431(7009):712-717.

57. Datta A, et al. Myc-ARF (alternate reading frame) interaction inhibits the functions of Myc. J Biol Chem. 2004;279(35):36698-36707.

58. Seoane J, Pouponnot C, Staller P, Schader M, Eilers M, Massague J. TGFbeta influences Myc, Miz-1 and Smad to control the CDK inhibitor p15INK4b. Nat Cell Biol. 2001;3(4):400-408.

59. Staller P, et al. Repression of $\mathrm{p} 15 \mathrm{INK} 4 \mathrm{~b}$ expression by Myc through association with Miz-1. Nat Cell Biol. 2001;3(4):392-399. 